\title{
Entropy Optimized Second Grade Fluid with MHD and Marangoni Convection Impacts: An Intelligent Neuro-Computing Paradigm
}

\author{
Muhammad Shoaib ${ }^{1}$ (D) Rafia Tabassum ${ }^{1}$, Kottakkaran Sooppy Nisar ${ }^{2, * \mathbb{D}}$, Muhammad Asif Zahoor Raja ${ }^{3, *(D)}$, \\ Ayesha Rafiq ${ }^{4}\left(\mathbb{D}\right.$, Muhammad Ijaz Khan ${ }^{5}$ (D), Wasim Jamshed ${ }^{6}$ (D), Abdel-Haleem Abdel-Aty ${ }^{7,8}$ (D) I. S. Yahia ${ }^{9,10,11}$ (D) \\ and Emad E. Mahmoud ${ }^{12}$ D
}

\section{check for} updates

Citation: Shoaib, M.; Tabassum, R.; Nisar, K.S.; Raja, M.A.Z.; Rafiq, A.; Khan, M.I.; Jamshed, W.; Abdel-Aty, A.-H.; Yahia, I.S.; E. Mahmoud, E. Entropy Optimized Second Grade Fluid with MHD and Marangoni Convection Impacts: An Intelligent Neuro-Computing Paradigm. Coatings 2021, 11, 1492. https:// doi.org/10.3390/coatings11121492

Academic Editor: Rahmat Ellahi

Received: 31 October 2021

Accepted: 28 November 2021

Published: 3 December 2021

Publisher's Note: MDPI stays neutral with regard to jurisdictional claims in published maps and institutional affiliations.

Copyright: (c) 2021 by the authors. Licensee MDPI, Basel, Switzerland. This article is an open access article distributed under the terms and conditions of the Creative Commons Attribution (CC BY) license (https:/ / creativecommons.org/licenses/by/ $4.0 /)$.
1 Department of Mathematics, COMSATS University Islamabad, Attock Campus, Punjab 43600, Pakistan; dr.shoaib@cuiatk.edu.pk (M.S.); sp20-rmt-006@cuiatk.edu.pk (R.T.)

2 Department of Mathematics, College of Arts and Sciences, Prince Sattam Bin Abdulaziz University, Wadi Aldawaser 11991, Saudi Arabia

3 Future Technology Research Center, National Yunlin University of Science and Technology, 123 University Road, Section 3, Yunlin 64002, Taiwan

4 Department of Applied Mathematics and Statistics, Institute of Space Technology, Islamabad 44000, Pakistan; ayesha.rafiq@mail.ist.edu.pk

5 Department of Mathematics and Statistic, Riphah International University, Islamabad 46000, Pakistan; mikhan@math.qau.edu.pk

6 Department of Mathematics, Capital University of Science and Technology (CUST), Islamabad 44000, Pakistan; wasiktk@hotmail.com

7 Department of Physics, College of Sciences, University of Bisha, Bisha 61922, Saudi Arabia; amabdelaty@ub.edu.sa

8 Physics Department, Faculty of Science, Al-Azhar University, Assiut 71524, Egypt

9 Laboratory of Nano-Smart Materials for Science and Technology (LNSMST), Department of Physics, Faculty of Science, King Khalid University, Abha 61413, Saudi Arabia; isyahia@gmail.com

10 Research Center for Advanced Materials Science (RCAMS), King Khalid University, Abha 61413, Saudi Arabia

11 Nanoscience Laboratory for Environmental and Biomedical Applications (NLEBA), Semiconductor Lab, Department of Physics, Faculty of Education, Ain Shams University, Cairo 11757, Egypt

12 Department of Mathematics and Statistics, College of Science, Taif University, Taif 21944, Saudi Arabia; e.mahmoud@tu.edu.sa

* Correspondence: n.sooppy@psau.edu.sa (K.S.N.); rajamaz@yuntech.edu.tw (M.A.Z.R.)

Abstract: Artificial intelligence applications based on soft computing and machine learning algorithms have recently become the focus of researchers' attention due to their robustness, precise modeling, simulation, and efficient assessment. The presented work aims to provide an innovative application of Levenberg Marquardt Technique with Artificial Back Propagated Neural Networks (LMT-ABPNN) to examine the entropy generation in Marangoni convection Magnetohydrodynamic Second Grade Fluidic flow model (MHD-SGFM) with Joule heating and dissipation impact. The PDEs describing MHD-SGFM are reduced into ODEs by appropriate transformation. The dataset is determined through Homotopy Analysis Method by the variation of physical parameters for all scenarios of proposed LMT-ABPNN. The reference data samples for training/validation/testing processes are utilized as targets to determine the approximated solution of proposed LMT-ABPNN. The performance of LMT-ABPNN is validated by MSE based fitness, error histogram scrutiny, and regression analysis. Furthermore, the influence of pertinent parameters on temperature, concentration, velocity, entropy generation, and Bejan number is also deliberated. The study reveals that the larger $\beta$ and $M a$, the higher $f^{\prime}(\eta)$ while $M$ has the reverse influence on $f^{\prime}(\eta)$. For higher values of $\beta, M, M a$, and $E c, \theta(\eta)$ boosts. The concentration $\phi(\eta)$ drops as Ma and Sc grow. An augmentation is noticed for $N_{G}$ for higher estimations of $\beta, M$, and $B r$. Larger $\beta, M$ and $B r$ decays the Bejan number.

Keywords: Levenberg Marquardt technique with artificial back propagated neural networks; homotopy analysis method; magnetohydrodynamic; second grade fluid; entropy optimization 


\section{Introduction}

The Marangoni boundary layer is a liquid-gas or liquid-liquid interface that leads to a dissipative layer on the fluid's surface. Many practical and industrial activities, such as aerospace engineering, chemical engineering, crystal formation, silicon melts, and liquid films, are driven either by the surface temperature gradient or surface concentration gradient. The thermal Marangoni effect and the solute Marangoni effect are the two types. Pearson [1] invented the former, while Scriven and Sternling [2] introduced the latter. When a liquid is heated from below, its surface temperature changes, according to Pearson's definition. This difference in surface temperature creates surface traction, which aids fluid flow in maintaining the temperature variation. As a result, a thermal Marangoni mechanism is created. The concentration of nanoparticles plays a role in the solute Marangoni effect by causing a surface concentration change, which leads to surface traction, which aids fluid motion. As a result, a solute Marangoni mechanism is created. This phenomenon has been demonstrated in numerous studies. Qayyum [3] attended the mechanism of Marangoni convection (MC) effect on hybrid nanofluid (HNF) by considering magnetohydrodynamic influence. Marangoni convection is used to study MHD flow of boundary layer Darcy-Forchheimer nanoliquid under effects of soret and dufour by Jawad et al. [4]. The thin film nanofluid stream and heat transmission scrutiny under combined effect of MC and MHD is explored by Gul et al. [5]. The combined effect of $\mathrm{MC}$ and magnetic field across thin liquid sheet of casson liquid, blood-based CNT nanoliquid is investigated by Akbar et al. [6]. Rasool et al. [7] conferred MC type casson nanoliquid stream in the existence of Lorentz forces subjected to Riga sheet.

The investigation of electrically conducting nanofluid flows subjected to magnetic fields has a wide range of applications, including nuclear reactors cooling, power generators, electrostatic filters and plasma, and geothermal energy exploitation. Rasool et al. [8] exemplified entropy production on MHD Darcy-Forchheimer nanoliquid stream past a non-linear stretchable sheet. Modather and Chamkha [9] analyzed the MHD micropolar fluid past vertical surface with transverse magnetic field. Krishna and Chamkha [10] conferred the impact of ion slip and Hall on rotating stream of nanofluid with MHD and diffusion-thermo effect. Boundary layer flow subjected to solar radiation past a flat inclined surface is investigated by Issa et al. [11]. Chamkha [12] considered the electrically conducting non-Darcy mixed convection stream within permeable medium in existence of hydromagnetic influence. Takhar et al. [13] exemplified the free stream velocity and Hall current on viscous fluid past a moveable surface with magnetohydrodynamic effect. Time dependent flow under magnetic field and heat transmission impact on semi-infinite flat surface is investigated by Nath et al. [14]. Chamkha [15] also discussed MHD natural convection stream with heat sink/source effect past a stretching sheet. Using finite element technique, Ali et al. [16] examined MHD micropolar liquid under thermal radiation and suction/injection due to inclined sheet. Habib et al. [17] numerically explored the time dependent MHD stream of Maxwell/tangent hyperbolic fluid over a two directional stretchable surface. The MHD stream of $\mathrm{Ag}-\mathrm{H}_{2} \mathrm{O}$ based nanoparticles with Corlios and Lorentz forces subjected to stretchable surface is debriefed by Siddique et al. [18].

Recently, some effective energy methods have been presented to reduce the amount of wasted energy. Entropy optimization (EOP) analysis is a useful tool for analyzing the optimization of various energy systems. When the irreversibilities are minimized, the optimal design of energy systems can be attained. The use of both the first and second laws of thermodynamics is used in this analysis. Entropy is generated for a variety of reasons, including heat exchange, body motion, mixing and expansion of substances, solid deformation, fluid flow, or any irreversible thermodynamic cycle, like heat pumps, heat engines, air conditioners, and power plants. Various research studies employ the EOP analysis. Khan et al. [19] analyzed the EOP of Darcy-Forchheimer stream on Hybrid nanoliquid considering Marangoni convection (MC) and homogeneous heterogeneous reaction. Kumar et al. [20] investigated the MC stream of HNF past a Darcy-Forchheimer media with EOP analysis. Qayyum et al. [21] explained the EOP under MC and irregular 
heat generation phenomenon subjected to rotating disk. The EOP in MHD stream of non-Newtonian fluid with MC and viscous dissipation effect is debriefed by Malaikah and Ijaz [22]. Marangoni bi-convection of non-Newtonian liquid subjected to inclined non Darcy medium with EOP by applying finite volume method (FVM) is investigated by Ahmed et al. [23]. Khan et al. numerically analyzed EOP and activation energy impact on MC stream of nanoliquid [24]. Hayat et al. [25] studied MHD stream of second grade fluid under impact of $\mathrm{MC}$ and EOP.

Artificial neural network (ANN) entrusts data processing to a large number of small and numerous processors that serve as interconnected and parallel ANNs. Pattern classification and recognition, regression, modeling, and mapping in various disciplines of bioinformatics, system control, temporal data forecasting, and other applications of ANN can be found. ANN is a burgeoning science and one of the most important trends in artificial intelligence. ANNs have been utilized to anticipate and develop diverse systems in recent years due to higher laboratory expenses as well as time savings [26-31]. Back propagation (BP) is a supervised machine learning solution to decrease network error via traversing the gradient of the error curve using the gradient descent method. In 1974, Paul Werbos developed the BP method. The Levenberg-Marquardt (LM) approach is an innovative convergent stability strategy for ANNs that can solve a broad range of fluid flow problems numerically. The Levenberg Marquardt technique with artificial back propagated neural network (LMT-ABPNN) was utilized by Khan et al. [32] to analyze the heat transmission of nanoliquid flow between two parallel sheets considering Brownian and thermophoretic phenomenon. Aljohani et al. [33] developed a wire coating model implementing LMT-ABPNN for Eyring Powell liquid under various effects. The Cross magneto nanoliquid stream in existence of magnetic force and permeable cylinder is modeled by Shah et al. [34] using LMT-ABPNN.

According to the studies cited above, intelligence computations based on ANN are still not investigated for EOP in MHD-SGFM with Marangoni convection, Joule heating, and dissipation impact. Furthermore, these stochastic techniques have numerous advantages over traditional techniques, and researchers are increasingly interested in using them in a variety of fields, including EOP [35,36], hybrid nanofluid models [37,38], magnetohydrodynamic flow model [39], functionally graded material [40], Falkner Skan Model [41], and numerous others [42-44]. Authors are motivated to examine the intelligent neurocomputing paradigm for numerical treatment and analysis of EOP in MHD-SGFM after reviewing these motivational recent relevant and valuable reported articles.

The following are some of the novel concepts concerning the suggested problem and soft computational model that are presented throughout this research study:

- An innovative application of Levenberg Marquardt Technique with artificial back propagated neural networks (LMT-ABPNN) is designed to examine the EOP in MHDSGFM with Marangoni convection, Joule heating, and dissipation impact.

- Generating datasets through HAM and utilizing in training/validation/testing processes as targets to determine the approximated solution of proposed LMT-ABPNN.

- The suggested technique efficiently examines the dynamics of the problem for many scenarios based on the variation of pertinent parameters to depict flows, velocity, concentration, and temperature profiles.

- The LMT-ABPNN's validity and verification are based on a thorough examination of accuracy assessments, histograms, and regression analysis conducted for the MHDSGFM, which are given graphically and numerically in sufficient detail.

The research paper's remaining structure is as follows: Section 2 provides the statement of the proposed MHD-SGFM. Sections 3 and 4 provide a detailed numerical and graphical description of the results obtained using the suggested LMT-ABPNN solver. Section 5 looks at temperature, concentration, and velocity including Absolute Error Examination, as well as the analysis of Bejan number and entropy optimization in MHD-SGFM. Section 6 contains the conclusions and future works. 


\section{Problem Statement}

Entropy-optimized magnetohydrodynamic flow of second-grade fluid is examined here. Temperature and concentration gradients cause flow to develop. The temperature gradient, magnetic field, and concentration gradient are used to compute Marangoni forced convection's dependency on surface pressure difference. In the influence of dissipation and Joule heating processes, the heat equation is discussed. The Marangoni effect is used to control the forward movement of liquid. The second law of thermodynamics is used to generate the physical attribute of irreversibility exploration. Consider that the $x$-axis lies parallel to the surface and the $y$-axis is perpendicular to it. At the surface, a constant magnetic field $B_{0}$ is applied. Assuming magnetic Reynolds number is small for flow, the induced magnetic field is ignored [45]. Moreover, no electric field is expected so the Hall effect is also neglected. Due to the non-existence of electric field small Reynolds number assumptions uncouple Maxwell conditions from Navier Stokes equations [46]. The temperature and concentration at the interface are supposed to be quadratic functions of the distance $x$ across it. The Marangoni surface tension effect, unlike the Boussinesq impact on the body force term in buoyancy-induced flows, serves as a boundary condition on the flow field's governing equations. The physical diagram is shown in Figure 1.

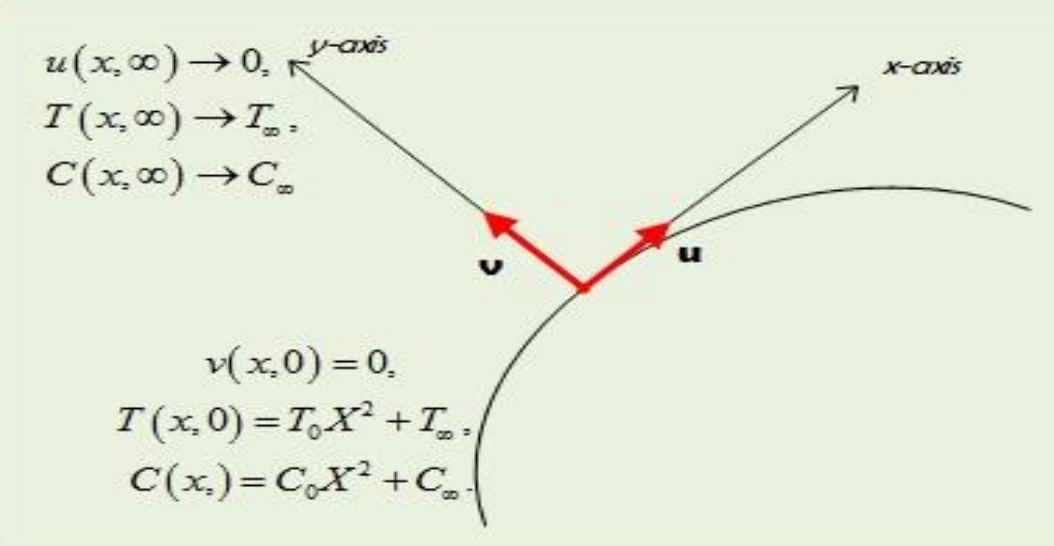

Figure 1. Flow geometry of the fluid flow system MHD-SGFM.

The following are the governing equations with B.Cs after applying boundary layer approximations [47-49];

$$
\begin{gathered}
\frac{\partial u}{\partial x}+\frac{\partial v}{\partial y}=0 \\
\frac{\partial u}{\partial x} u+\frac{\partial u}{\partial y} v=\frac{\partial^{2} u}{\partial y^{2}} v+\left(\frac{\partial^{2} u}{\partial y^{2}} \frac{\partial u}{\partial x}-\frac{\partial^{2} u}{\partial x \partial y} \frac{\partial u}{\partial y}+\frac{\partial^{3} u}{\partial x \partial y^{2}} u+\frac{\partial^{3} u}{\partial y^{3}} v\right) \alpha_{1}-u \frac{B_{0}^{2} \sigma_{1}}{\rho} \\
\frac{\partial T}{\partial x} u+\frac{\partial T}{\partial y} v=\frac{\partial^{2} T}{\partial y^{2}} \alpha+\frac{1}{\left(\rho c_{p}\right)}\left(\left(\frac{\partial u}{\partial y}\right)^{2} \mu+\left(\frac{\partial^{2} u}{\partial x \partial y} \frac{\partial u}{\partial y} u+\frac{\partial^{2} u}{\partial y^{2}} \frac{\partial u}{\partial y} v\right) \alpha_{1}+u^{2} B_{0}^{2} \sigma_{1}\right) \\
\frac{\partial C}{\partial x} u+\frac{\partial C}{\partial y} v=\frac{\partial^{2} C}{\partial y^{2}} D_{B}, \\
\mu \frac{\partial u}{\partial y}=-\frac{\partial \sigma}{\partial x}=\left(\frac{\partial T}{\partial x} \gamma_{T}+\frac{\partial C}{\partial x} \gamma_{C}\right) \sigma_{0}, v=0, T=T_{0} X^{2}+T_{\infty}, C=C_{0} X^{2}+C_{\infty}, \text { at } y=0, \\
u(x, \infty) \rightarrow 0, T(x, \infty) \rightarrow T_{\infty}, C(x, \infty) \rightarrow C_{\infty}, X=\frac{x}{L_{1}}, \text { at } y=\infty
\end{gathered}
$$

Surface tension defined as the linear function of concentration and temperature:

$$
\sigma=\sigma_{0}-\left(C-C_{\infty}\right) \gamma_{C}-\left(T-T_{\infty}\right) \gamma_{T}
$$


With

$$
\gamma_{C}=-\left.\frac{\partial \sigma}{\partial C}\right|_{C=C_{\infty}}, \gamma_{T}=-\left.\frac{\partial \sigma}{\partial T}\right|_{T=T_{\infty}} .
$$

Consider $[46,47]$

$$
\begin{gathered}
\psi=v X f(\eta), \eta=\frac{y}{L_{1}}, u=\frac{\partial \psi}{\partial y}, v=-\frac{\partial \psi}{\partial x} \\
T=\theta(\eta) T_{0} X^{2}+T_{\infty}, C=\phi(\eta) C_{0} X^{2}+C_{\infty}
\end{gathered}
$$

Applying above suitable similarity transformation on PDEs system (1-5) we get ODEs with B.Cs as follows:

$$
\begin{gathered}
f^{\prime \prime \prime}-f^{\prime 2}+f f^{\prime \prime}-M f^{\prime}+\left(2 f^{\prime \prime \prime} f^{\prime}-f^{\prime \prime 2}-f f^{i v}\right) \beta=0, \\
\theta^{\prime \prime}-2 f^{\prime} \theta \operatorname{Pr}+\theta^{\prime} f \operatorname{Pr}+f^{\prime \prime 2} E c \operatorname{Pr}+f^{\prime 2} M \operatorname{Pr} E c+\left(f^{\prime \prime} f^{\prime}-f^{\prime \prime \prime} f^{\prime \prime} f\right) \operatorname{Pr} \beta E c=0, \\
\phi^{\prime \prime}-2 f^{\prime} \phi S c+f \phi^{\prime} S c=0, \\
f(0)=0, f^{\prime \prime}(0)=-2+2 M a, \theta(0)=1, \phi(0)=1, \\
f^{\prime}(\infty)=0, \theta(\infty)=0, \phi(\infty)=0 .
\end{gathered}
$$

Here second grade fluid parameter, Hartmann number, Prandtl Number, Schmidt Number, Marangoni ratio parameter, and Eckert number are mathematically expressed as $\beta=\frac{\alpha_{1}}{L_{1}^{2} \rho}, M=\frac{L_{1}^{2} B_{0}^{2} \sigma_{1}}{\mu}, \operatorname{Pr}=\frac{v}{\alpha}, S c=\frac{v}{D}, M a=\frac{C_{0} \gamma_{C}}{T_{0} \gamma_{T}}, E c=\frac{v^{2}}{c_{p} T_{0} L_{1}^{2}}$ respectively.

\section{Entropy Optimization}

The following is dimensional form of entropy optimization $\left(N_{G}\right)$ :

$$
\begin{aligned}
S_{G}=\left(\frac{\partial T}{\partial y}\right)^{2} \frac{k}{T_{0}^{2}}+ & \left(\frac{\partial^{2} u}{\partial x \partial y} \frac{\partial u}{\partial y} u+\frac{\partial^{2} u}{\partial y^{2}} \frac{\partial u}{\partial y} v\right) \frac{\alpha_{1}}{T_{0}}+\left(\frac{\partial u}{\partial y}\right)^{2} \frac{\mu}{T_{0}}+\frac{u^{2} B_{0}^{2} \sigma}{T_{0}} \\
& +\left(\frac{\partial C}{\partial y}\right)^{2} \frac{R_{D}}{C_{0}}+\left(\frac{\partial C}{\partial y} \frac{\partial T}{\partial y}\right) \frac{R_{D}}{T_{0}}
\end{aligned},
$$

The dimensionless form is

$$
N_{G}={\theta^{\prime}}^{2}+f^{\prime \prime 2} \frac{B r}{A}+\left(f^{\prime \prime 2} f^{\prime}-f^{\prime \prime \prime} f^{\prime \prime} f\right) \frac{B r \beta}{A}+f^{\prime 2} M B r+\theta^{\prime} \phi^{\prime} L+\phi^{\prime 2} L .
$$

The Bejan number

$$
B e=\frac{\theta^{\prime 2}+\theta^{\prime} \phi^{\prime} L+\phi^{\prime 2} L}{N_{G}} .
$$

Mathematically, entropy rate, Brinkman number, diffusion parameter, and dimensionless parameter expressed as $N_{G}=\frac{L_{1}^{2} S_{G}}{X^{4} k}, B r=\frac{v^{2} \mu}{k L_{1}^{2} T_{0}}, L=\frac{C_{0} R_{D}}{k}, A=\frac{x}{L_{1}}$ respectively.

\section{Solution Methodology}

The brief discussion of results for proposed LMT-ABPNN subjected to entropy generation in Marangoni convection magnetohydrodynamic second grade fluidic flow model (MHD-SGFM) with Joule heating and dissipation impact has been analyzed in this section. The PDEs describing MHD-SGFM are reduced into ODEs by appropriate transformation. The dataset is determined through homotopy analysis method by the variation of second grade fluid parameter $(\beta)$, modified Hartmann number $(M)$, Marangoni ratio parameter $(M a)$, Eckert number $(E c)$, and Schmidt number $(S c)$. To solve governing ODEs that characterize the flow model in Equations (9)-(12), the LMT-ABPNN solver MATLAB integral toolbox for artificial neural networks (ANN) is utilized with the help of "nftool" command. LMT-ABPNN has a two-layer structure (single input, hidden, and output) for supervised neural networks using LMT backpropagation. As illustrated in 
Figure 2, the ANNs architecture is built on 10 neuron numbers with log-sigmoid activation, whereas Figure 3 depicts the process flow architecture. The reference data samples for training/validation/testing processes are utilized as target to determine the approximated solution of proposed LMT-ABPNN. The LMT-ABPNN's validity and verification are based on a thorough examination of accuracy assessments, histograms, and regression analysis conducted for the MHD-SGFM, which are given graphically and numerically in sufficient detail (See Table 1).

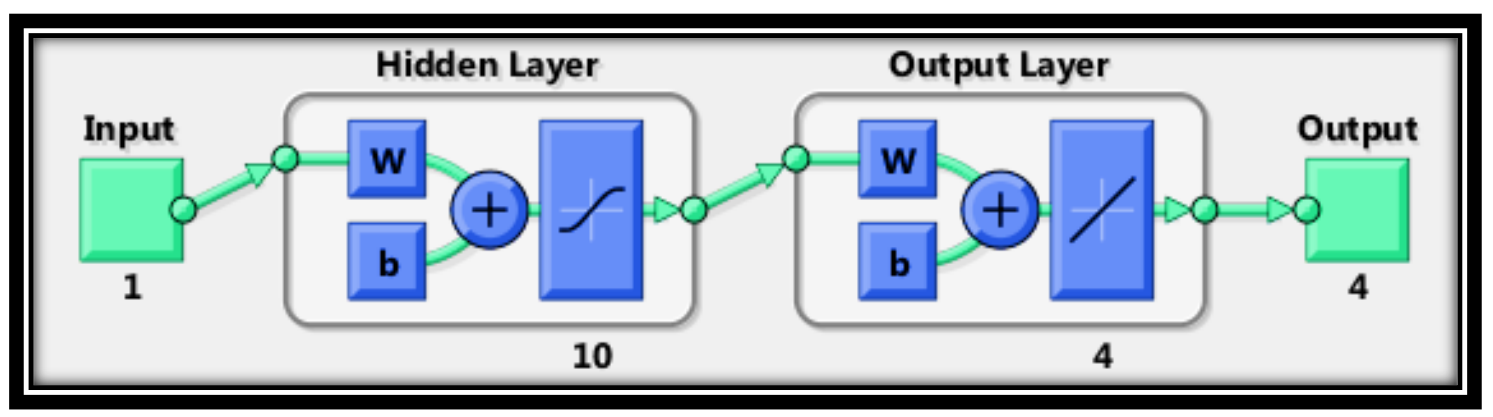

Figure 2. The Neural Network for MHD-SGFM.

Table 1. Variants of MHD-SGFM.

\begin{tabular}{cccccc}
\hline Scenario & Fluid Variants & Case (1) & Case (2) & Case (3) & Case (4) \\
\hline 1 & $\beta$ & 0.1 & 0.4 & 0.7 & 1.0 \\
\hline 2 & $M$ & 0.1 & 0.6 & 1.2 & 1.8 \\
\hline 3 & $M a$ & 0.1 & 0.4 & 0.7 & 1.0 \\
\hline 4 & $E c$ & 0.1 & 0.4 & 0.7 & 1.0 \\
\hline 5 & $S c$ & 0.1 & 0.3 & 0.5 & 0.7 \\
\hline
\end{tabular}

For all four cases of five different scenarios of LMT-BPNN of MHD-SGFM, the reference dataset for velocity, temperature, and concentration profiles of proposed LMT-BPNN is suggested using homotopy analysis method for $\eta$ between 0 and 5, with step size of 0.05 for all four cases of five different scenarios of LMT-ABPNN. In this paper, the obtained datasets are evaluated in terms of $f^{\prime}(\eta), \theta(\eta)$ and $\phi(\eta)$ as reference outcomes. Tables $2-4$ show the results of LMT-ABPNN for variants of $f^{\prime}(\eta), \theta(\eta)$, and $\phi(\eta)$ of MHDSGFM in terms of backpropagation networks, MSE (Training/validation/testing) and total iterations/epochs, and time taken for all the scenarios connected with MHD-SGFM.

Table 2. Outcomes of LMT-ABPNN for variants of $f^{\prime}(\eta)$ of MHD-SGFM.

\begin{tabular}{|c|c|c|c|c|c|c|c|c|}
\hline \multirow{2}{*}{$\begin{array}{c}\text { Physical } \\
\text { Quantities }\end{array}$} & \multicolumn{3}{|c|}{ MSE } & \multirow{2}{*}{ Performance } & \multirow{2}{*}{ Grad } & \multirow{2}{*}{ Mu } & \multirow{2}{*}{ Epochs } & \multirow{2}{*}{ Time } \\
\hline & Training & Validation & Testing & & & & & \\
\hline$\beta$ & $2.52 \times 10^{-9}$ & $5.56 \times 10^{-9}$ & $4.53 \times 10^{-9}$ & $2.52 \times 10^{-9}$ & $9.98 \times 10^{-8}$ & $1.00 \times 10^{-8}$ & 916 & $34 \mathrm{~s}$ \\
\hline$M$ & $2.14 \times 10^{-9}$ & $2.47 \times 10^{-9}$ & $2.41 \times 10^{-9}$ & $1.83 \times 10^{-9}$ & $1.55 \times 10^{-6}$ & $1.00 \times 10^{-9}$ & 565 & $11 \mathrm{~s}$ \\
\hline$M a$ & $1.01 \times 10^{-10}$ & $1.38 \times 10^{-10}$ & $1.46 \times 10^{-10}$ & $1.01 \times 10^{-10}$ & $2.96 \times 10^{-7}$ & $1.00 \times 10^{-10}$ & 1000 & $32 \mathrm{~s}$ \\
\hline
\end{tabular}




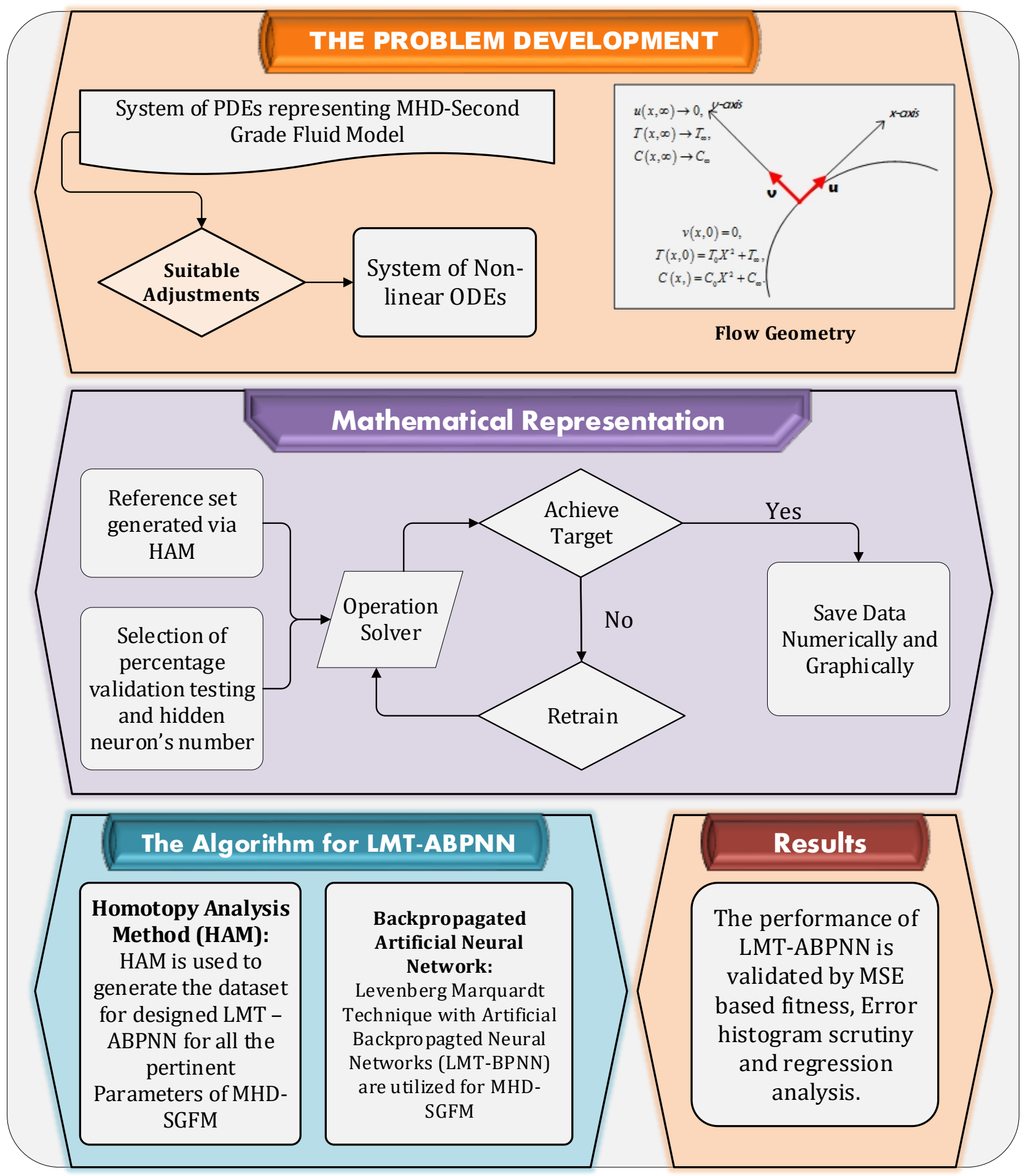

Figure 3. The Flow Architecture for MHD-SGFM. 
Table 3. Outcomes of LMT-ABPNN for variants of $\theta(\eta)$ of MHD-SGFM.

\begin{tabular}{|c|c|c|c|c|c|c|c|c|}
\hline \multirow{2}{*}{$\begin{array}{l}\text { Physical } \\
\text { Quantities }\end{array}$} & \multicolumn{3}{|c|}{ MSE } & \multirow{2}{*}{ Performance } & \multirow{2}{*}{ Grad } & \multirow{2}{*}{$\mathbf{M u}$} & \multirow{2}{*}{ Epochs } & \multirow{2}{*}{ Time } \\
\hline & Training & Validation & Testing & & & & & \\
\hline$\beta$ & $1.99 \times 10^{-10}$ & $\begin{array}{l}2.24 \times \\
10^{-10}\end{array}$ & $\begin{array}{l}9.02 \times \\
10^{-10}\end{array}$ & $1.99 \times 10^{-10}$ & $9.95 \times 10^{-8}$ & $1.00 \times 10^{-8}$ & 432 & $10 \mathrm{~s}$ \\
\hline$M$ & $2.99 \times 10^{-9}$ & $1.45 \times 10^{-9}$ & $4.09 \times 10^{-9}$ & $2.99 \times 10^{-9}$ & $9.90 \times 10^{-8}$ & $1.00 \times 10^{-8}$ & 508 & $24 \mathrm{~s}$ \\
\hline$M a$ & $5.69 \times 10^{-10}$ & $\begin{array}{c}9.50 \times \\
10^{-10}\end{array}$ & $2.79 \times 10^{-9}$ & $5.69 \times 10^{-10}$ & $9.94 \times 10^{-8}$ & $1.00 \times 10^{-8}$ & 312 & $9 \mathrm{~s}$ \\
\hline Ec & $2.01 \times 10^{-10}$ & $\begin{array}{l}3.03 \times \\
10^{-10}\end{array}$ & $\begin{array}{l}4.34 \times \\
10^{-10}\end{array}$ & $2.01 \times 10^{-10}$ & $9.95 \times 10^{-8}$ & $1.00 \times 10^{-8}$ & 379 & $14 \mathrm{~s}$ \\
\hline
\end{tabular}

Table 4. Outcomes of LMT-ABPNN for variants of $\phi(\eta)$ of MHD-SGFM.

\begin{tabular}{|c|c|c|c|c|c|c|c|c|}
\hline \multirow{2}{*}{$\begin{array}{c}\text { Physical } \\
\text { Quantities }\end{array}$} & \multicolumn{3}{|c|}{ MSE } & \multirow{2}{*}{ Performance } & \multirow{2}{*}{ Grad } & \multirow{2}{*}{$\mathbf{M u}$} & \multirow{2}{*}{ Epochs } & \multirow{2}{*}{ Time } \\
\hline & Training & Validation & Testing & & & & & \\
\hline$M a$ & $7.13 \times 10^{-10}$ & $1.81 \times 10^{-9}$ & $1.01 \times 10^{-9}$ & $7.13 \times 10^{-10}$ & $9.90 \times 10^{-8}$ & $1.00 \times 10^{-8}$ & 226 & $8 \mathrm{~s}$ \\
\hline Sc & $1.81 \times 10^{-9}$ & $3.91 \times 10^{-9}$ & $3.47 \times 10^{-9}$ & $1.81 \times 10^{-9}$ & $9.99 \times 10^{-8}$ & $1.00 \times 10^{-8}$ & 192 & $6 \mathrm{~s}$ \\
\hline
\end{tabular}

\section{Result Interpretations}

Figures 4a, 5a, 6a, 7a, 8a, 9a, 10a, 11a and 12a show graphs for designed LMTABPNN for MHD-SGFM representing convergence of validation, training, and testing process against epochs index for solving the cases of $\beta, M$, and $M a$ for $f^{\prime}(\eta)$; the cases of $\beta, M, M a$, and Ec for $\theta(\eta)$; and finally, the cases of $M a, S c$ for $\phi(\eta)$. The best excellent validation performance obtained at epochs 916, 559, 1000, 432, 508, 312, 379, 226, and 192 with MSE almost $5.56 \times 10^{-9}, 2.47 \times 10^{-9}, 1.38 \times 10^{-10}, 2.24 \times 10^{-10}, 1.45 \times 10^{-9}, 9.50 \times 10^{-10}$, $3.03 \times 10^{-10}, 1.81 \times 10^{-9}$ and $3.91 \times 10^{-9}$ in time $34,11,32,10,24,9,14,8$, and $6 \mathrm{~s}$ respectively. It illustrates that all lines are smooth and approaches to a same point which authenticates the ideal performance. The corresponding figures and Tables $2-4$ suggest, with lower MSE value the performance attitude will be best.

Figures $4 b, 5 b, 6 b, 7 b, 8 b, 9 b, 10 b, 11 b$ and $12 b$ reveal the convergent efficiency perfection and accuracy for solving the cases of $\beta, M$, and $M a$ for $f^{\prime}(\eta)$; the cases of $\beta, M, M a$, and Ec for $\theta(\eta)$; and finally, the cases of $M a, S_{c}$ for $\phi(\eta)$. The gradient values in all of these scenarios, as well as the values of the Mu parameter during training in identifying another vector, are shown in Figures $4 b, 5 b, 6 b, 7 b, 8 b, 9 b, 10 b, 11 b$ and $12 b$. $\mathrm{Mu}$ is the neural network training algorithm's adaptive parameter. Error convergence is directly influenced by the choice of $\mathrm{Mu}$. The associated values of gradient $5.56 \times 10^{-8}$, $1.55 \times 10^{-6}, 2.96 \times 10^{-7}, 9.95 \times 10^{-8}, 9.90 \times 10^{-8}, 9.94 \times 10^{-8}, 9.95 \times 10^{-8}, 9.90 \times 10^{-8}$ and $9.99 \times 10^{-8}$ with $\mathrm{Mu}$ parameter $10^{-8}, 10^{-9}, 10^{-10}, 10^{-8}, 10^{-8}, 10^{-8}, 10^{-8}, 10^{-8}$, and $10^{-8}$, respectively. Results reveal that a rise in epoch corresponds to a reduction in the gradient and $\mathrm{Mu}$ values. The extensive training of networks results in the form of better convergence of the solution with lowest $\mathrm{Mu}$ and gradient values.

Figures $4 c, 5 c, 6 c, 7 c, 8 c, 9 c, 10 c, 11 c$ and 12c depict graphical representations of the fitness analysis for the fluid flow highlighted variants associated with the mathematical model, and the error shows the difference between the targeted and reference solutions. For all scenarios, the graph shows that the target values overlap the output values of the proposed LMT-BPNN solver, which validates the accuracy of solution. 


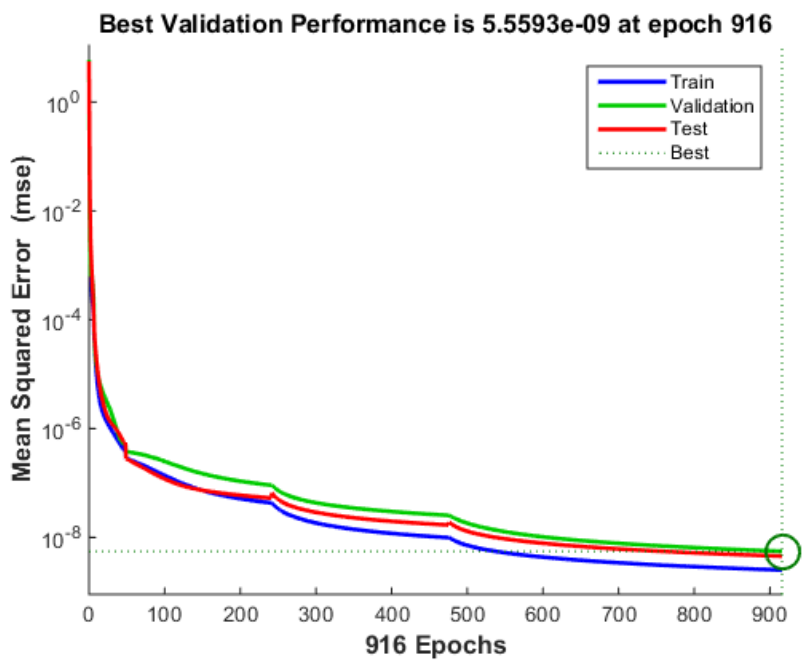

(a)

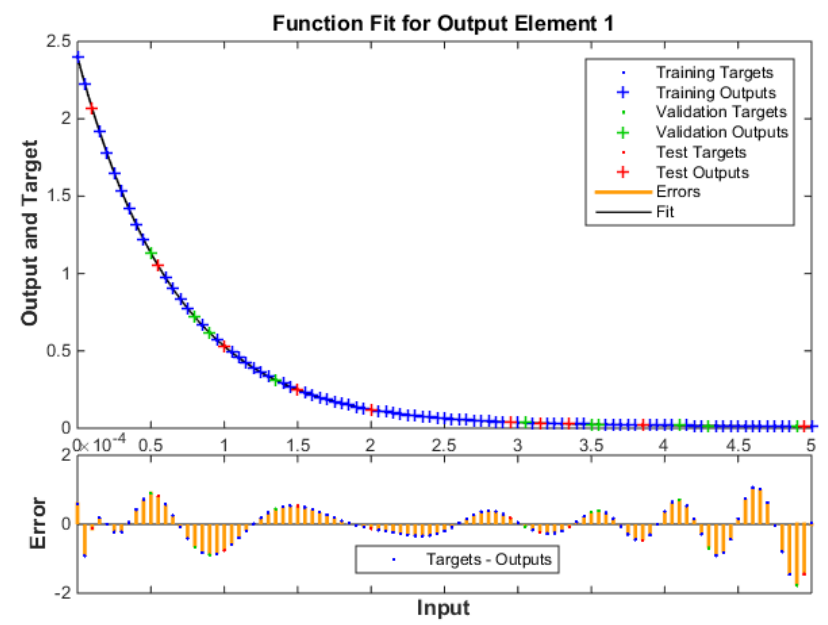

(c)
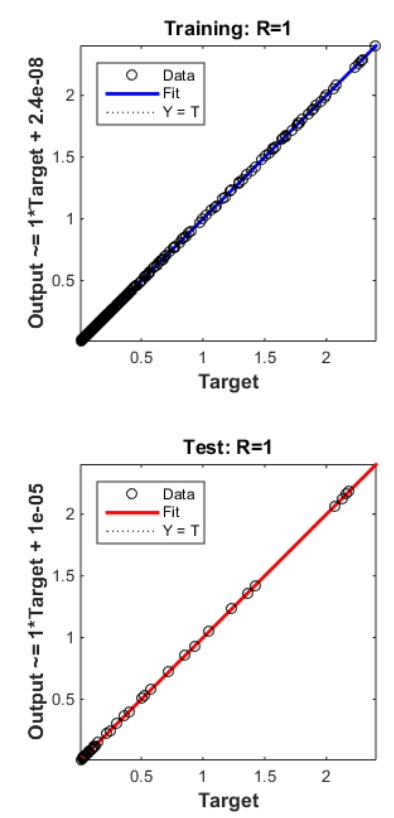

(e)

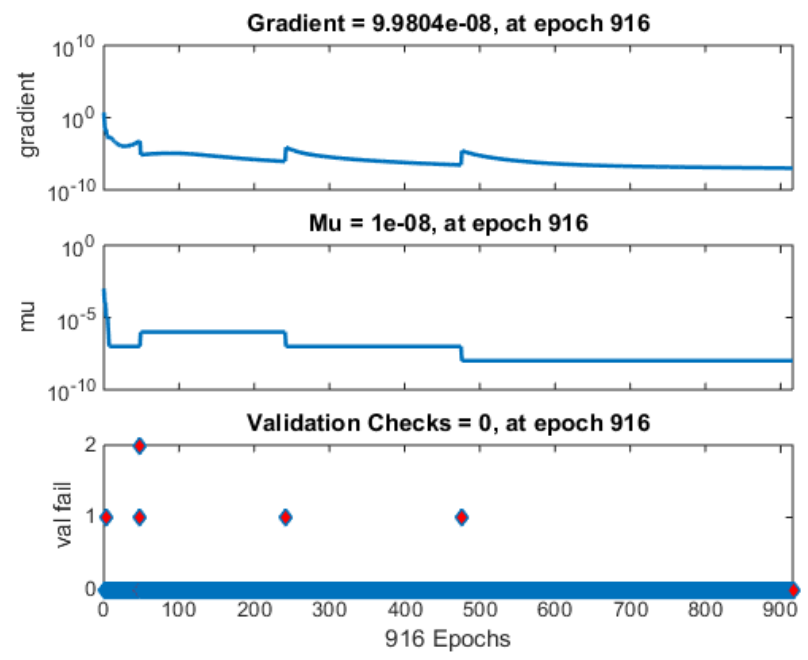

(b)

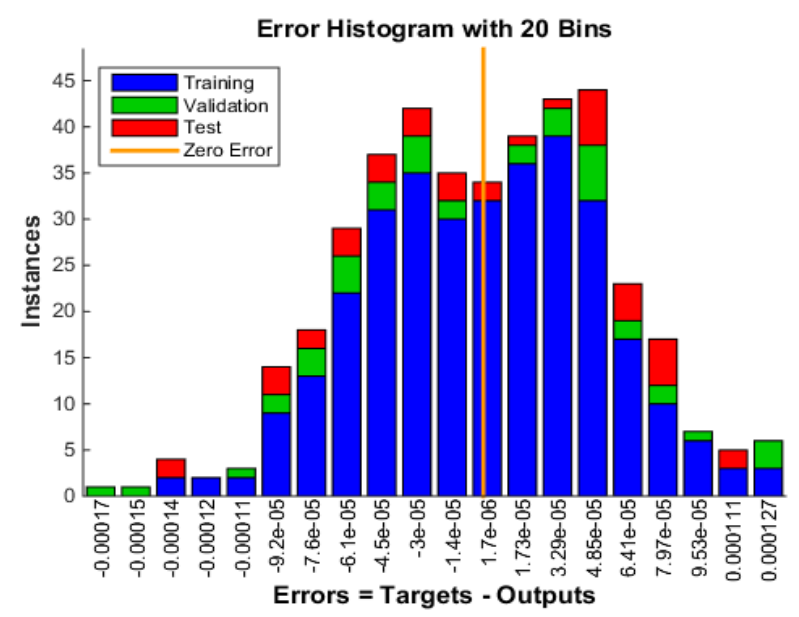

(d)
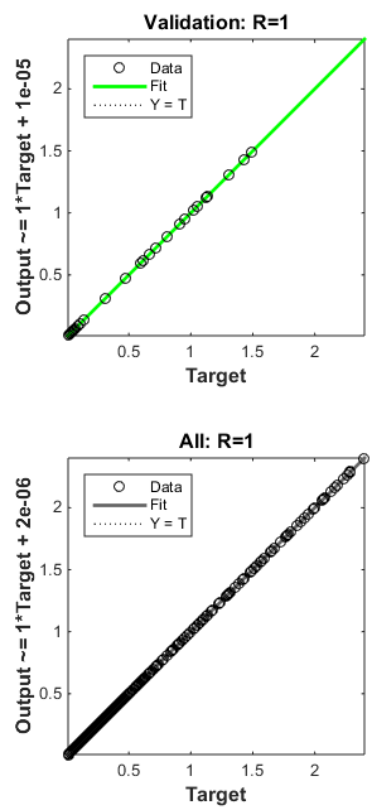

Figure 4. Graphic representation for LMT-ABPNN base on variants of $\beta$ for $f^{\prime}(\eta)$ of MHD-SGFM. (a) MSE representation, (b) Results of the transition state, (c) Fitness of the curve, (d) Histogram for error analysis, (e) Regression. 


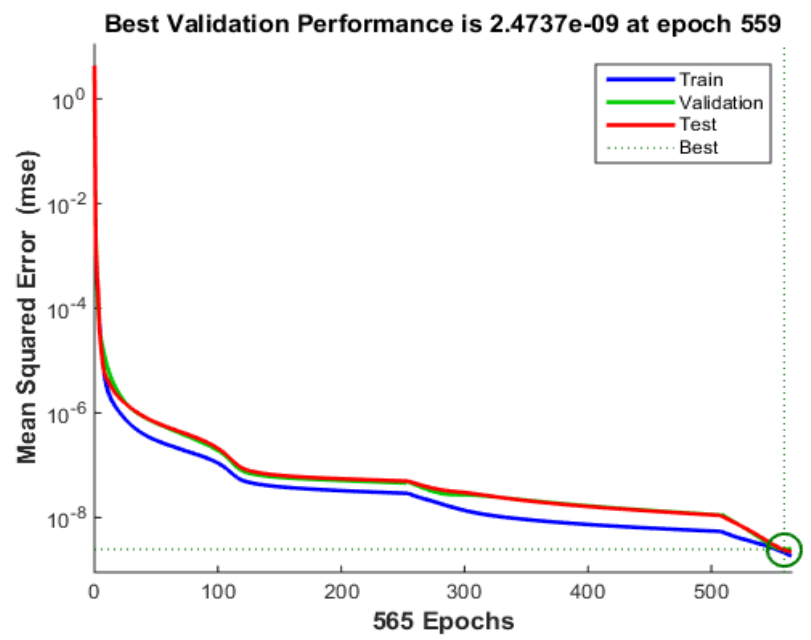

(a)

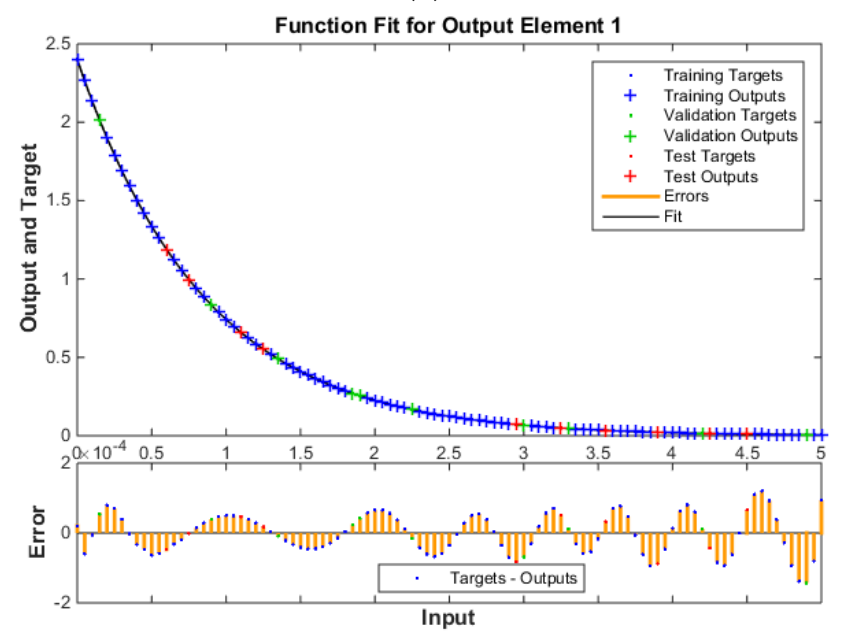

(c)
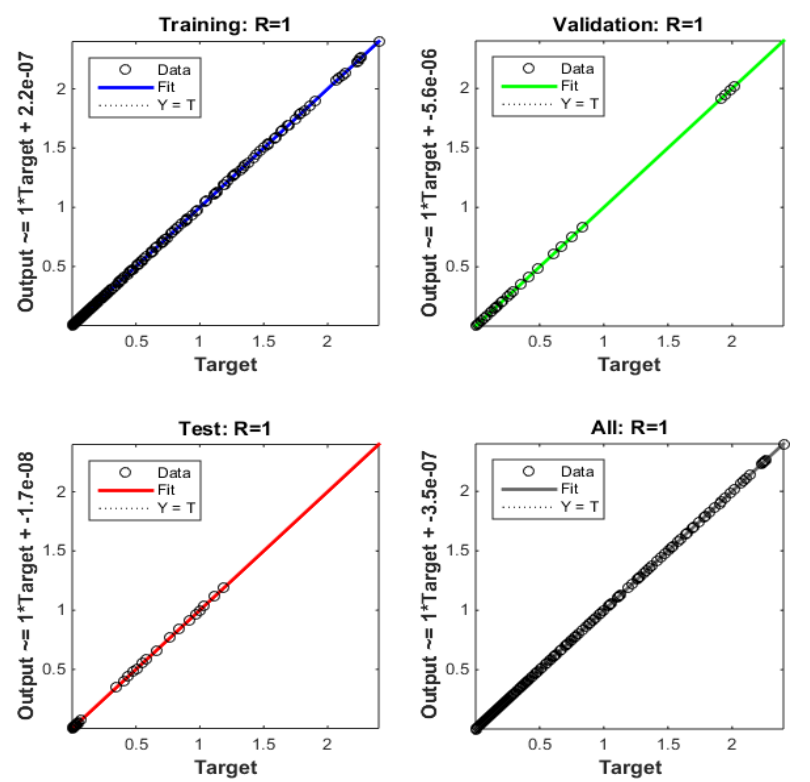

(e)

Figure 5. Graphic representation for designed LMT-ABPNN for solving cases of $M$ for $f^{\prime}(\eta)$ of MHD-SGFM. (a) MSE Result, (b) Results of the transition state, (c) Fitness of the curve, (d) Histogram for error analysis, (e) Regression.
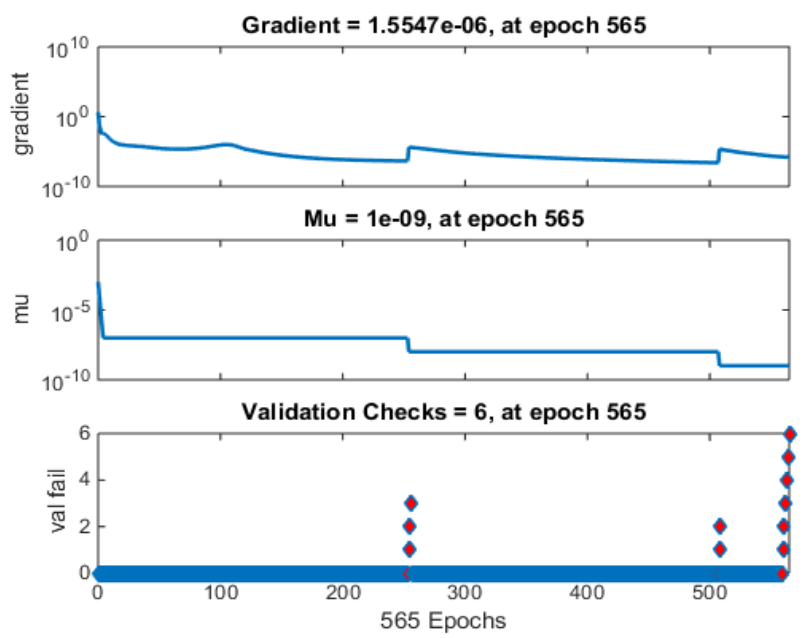

(b)

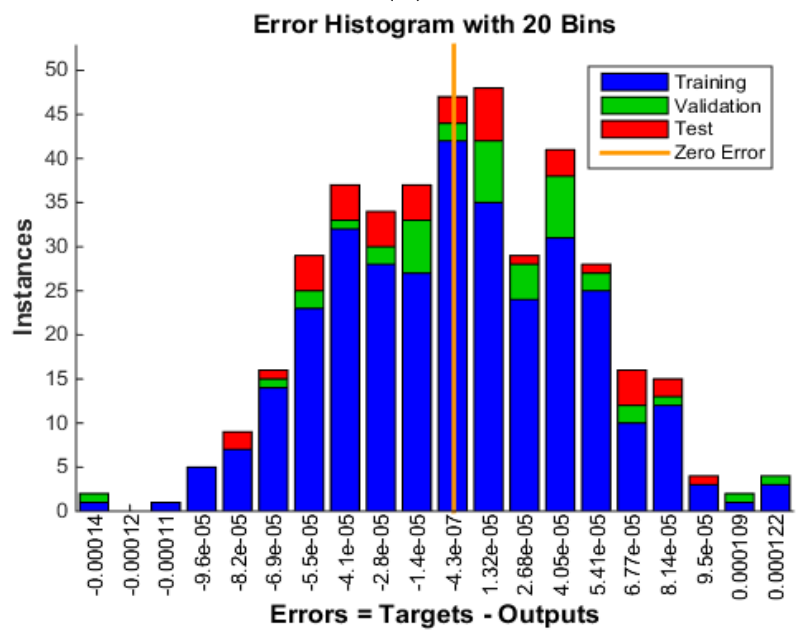

(d)

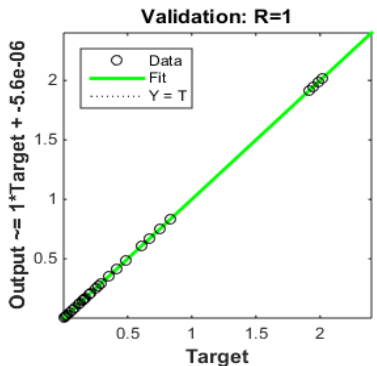




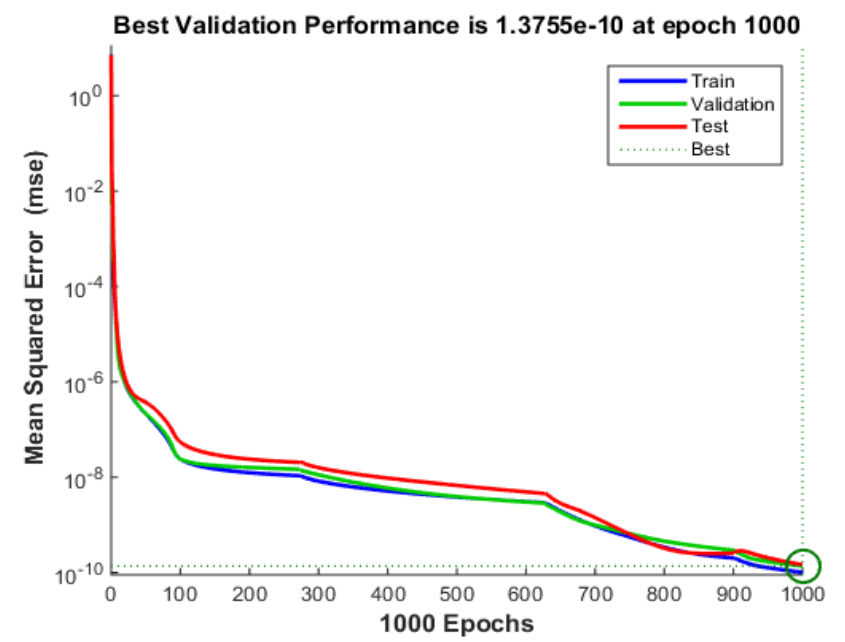

(a)

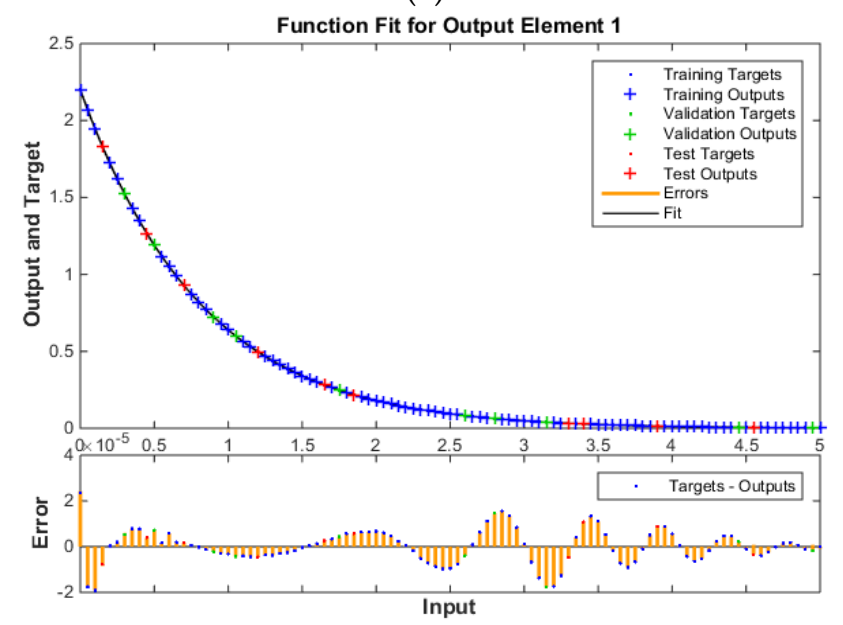

(c)
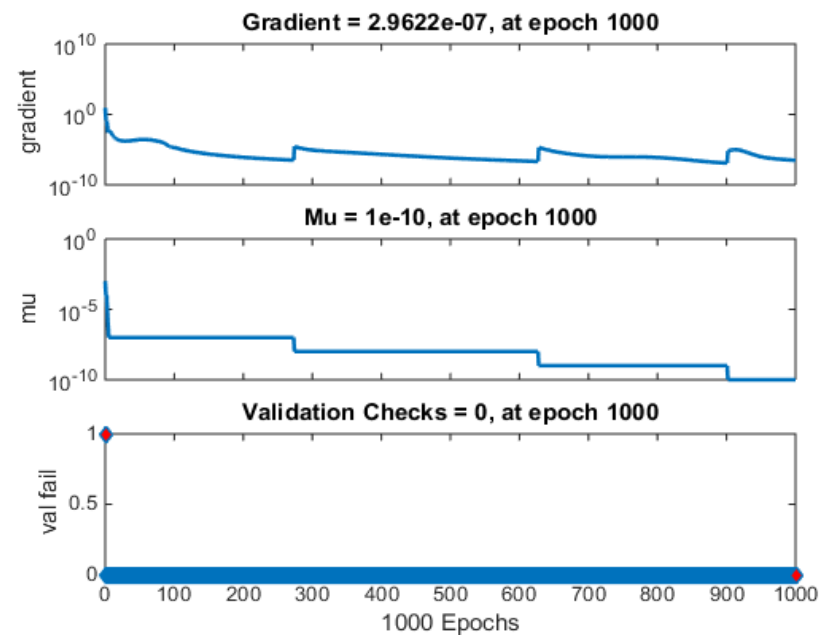

(b)

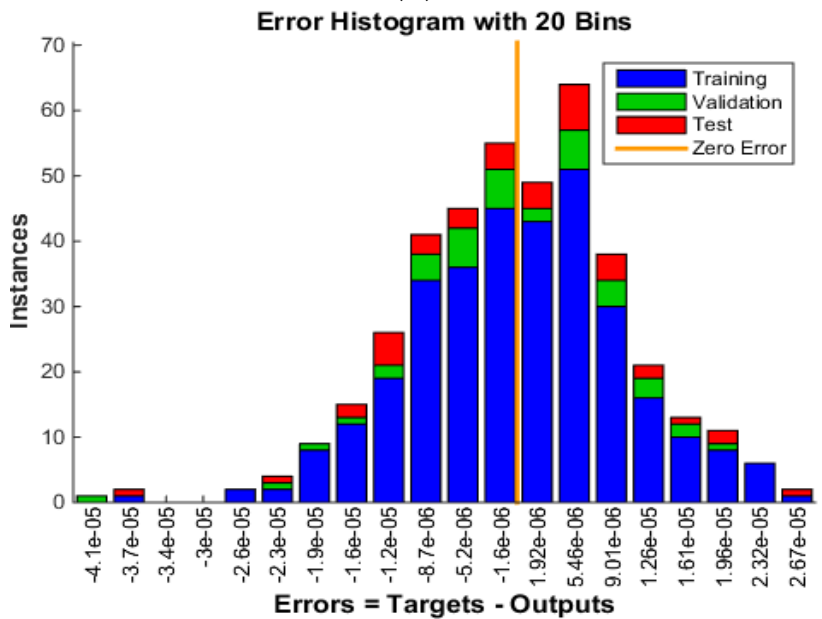

(d)
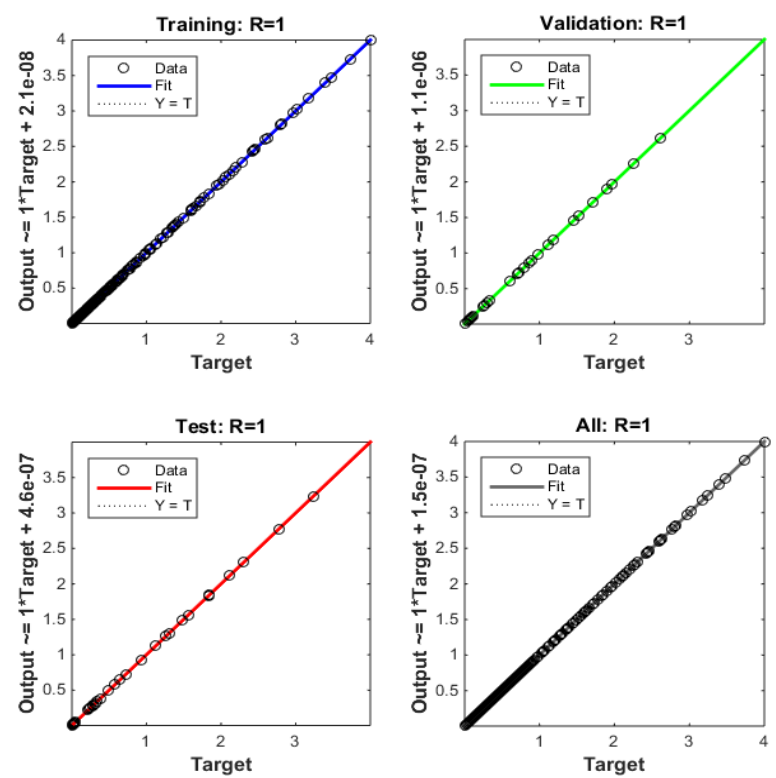

(e)

Figure 6. Graphic representation for designed LMT-ABPNN for solving cases of $M a$ for $f^{\prime}(\eta)$ of MHD-SGFM. (a) MSE Result, (b) Results of the transition state, (c) Fitness of the curve, (d) Histogram for error analysis, (e) Regression. 


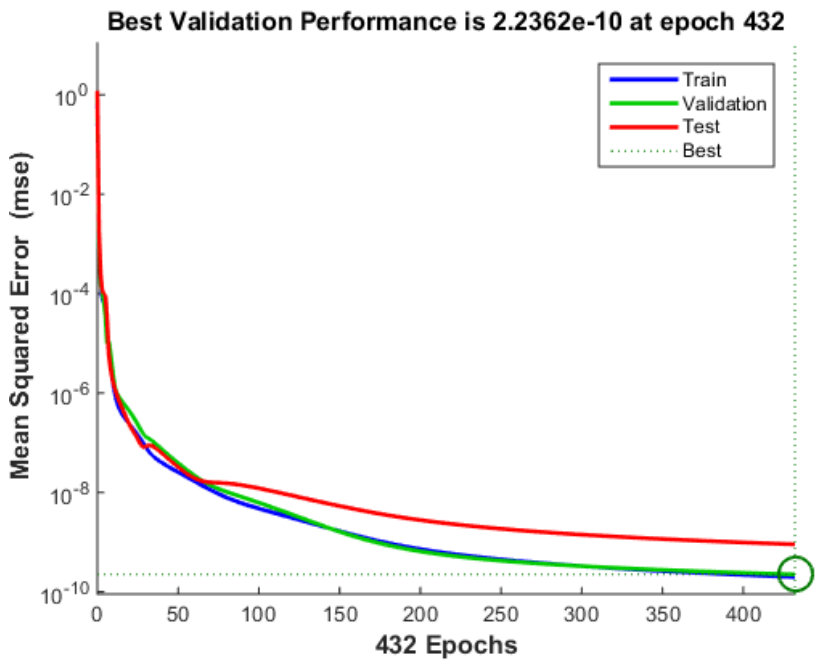

(a)

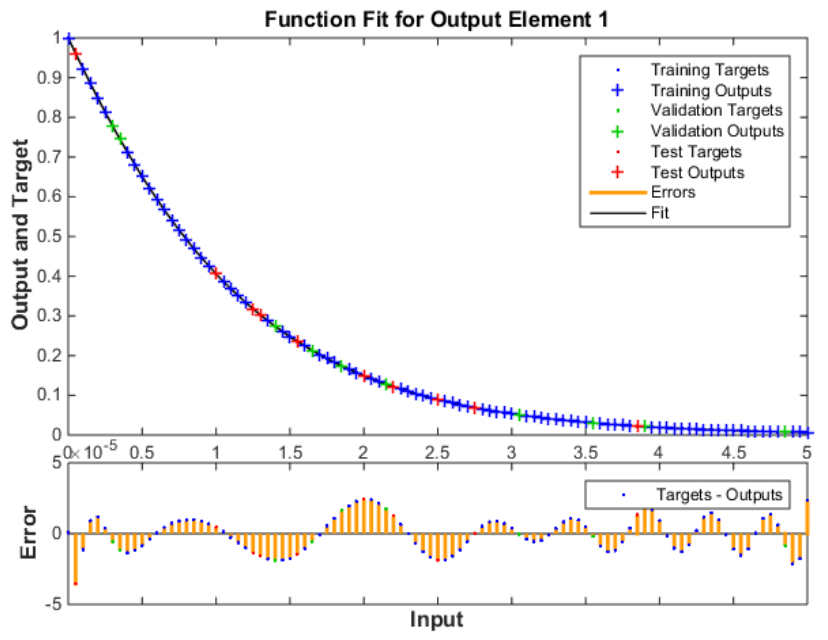

(c)
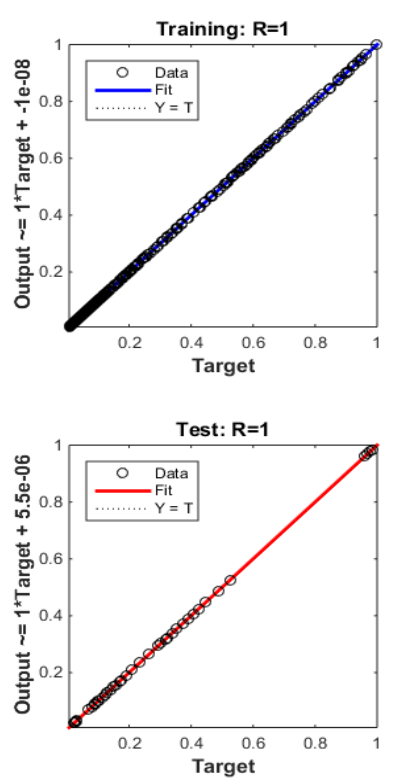

(e)

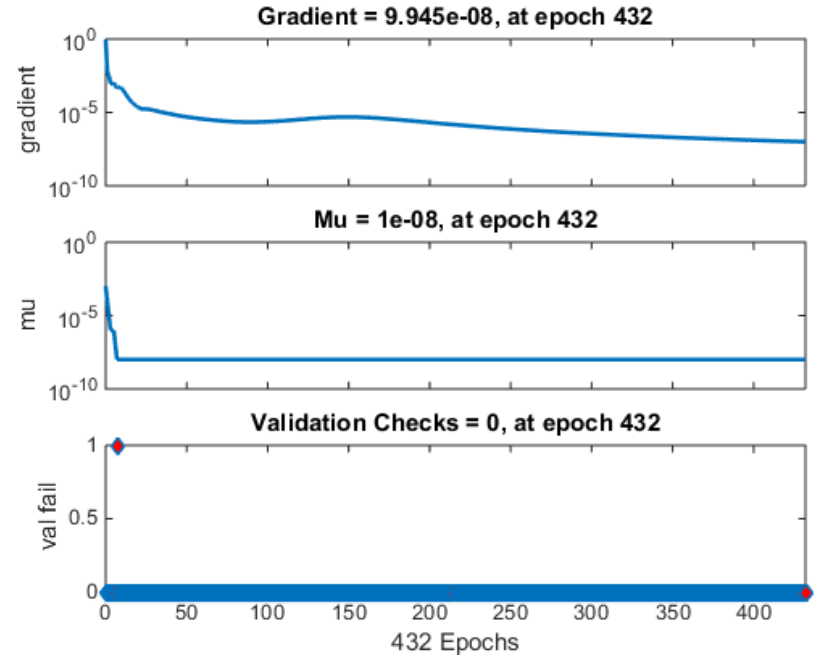

(b)

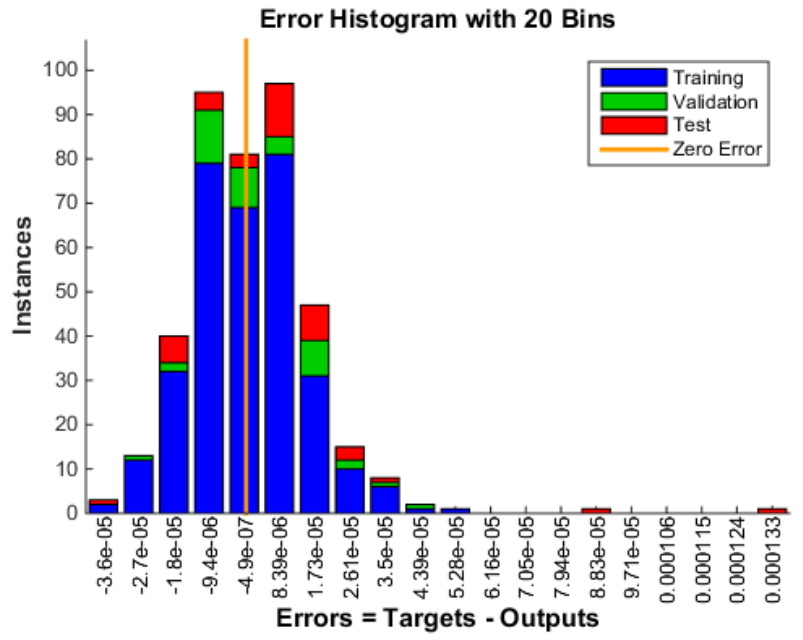

(d)
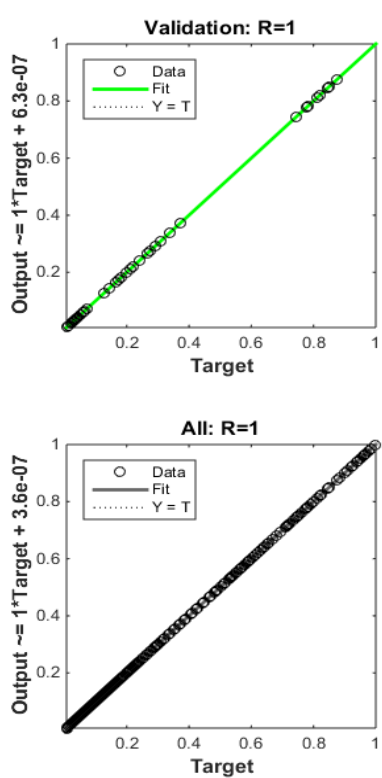

Figure 7. Graphic representation for designed LMT-ABPNN for solving cases of $\beta$ for $\theta(\eta)$ of MHD-SGFM. (a) MSE Result, (b) Results of the transition state, (c) Fitness of the curve, (d) Histogram for error analysis, (e) Regression. 


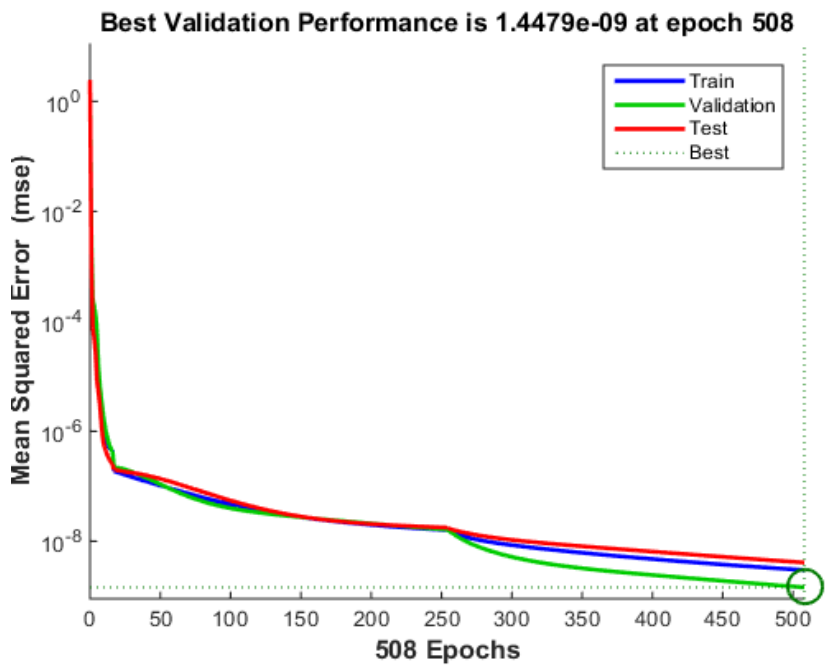

(a)

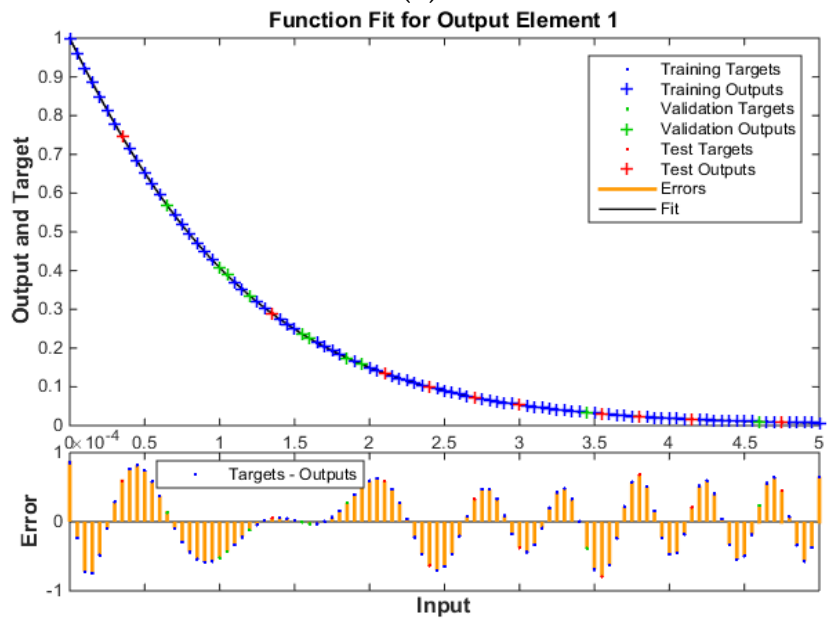

(c)
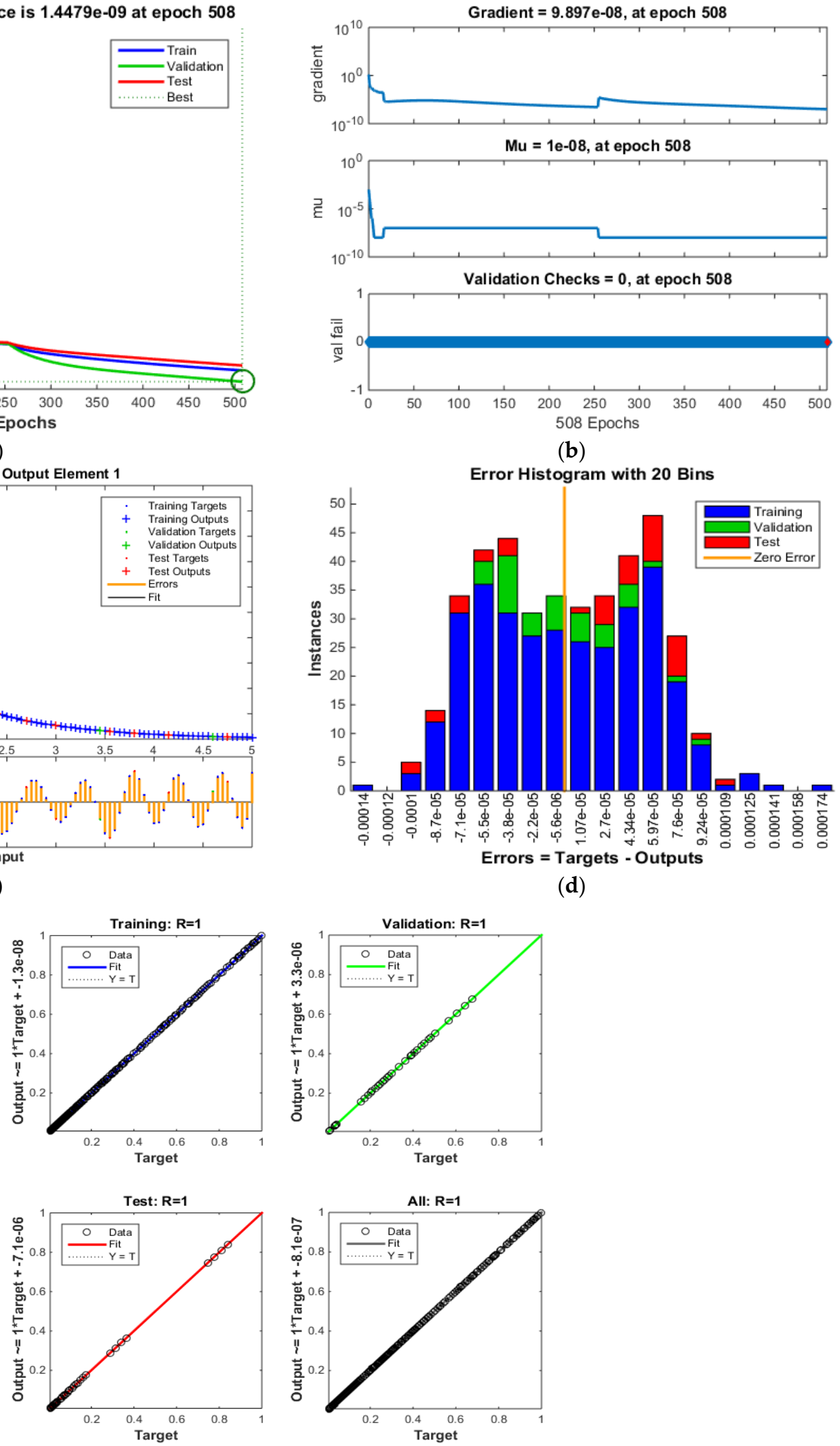

(b)

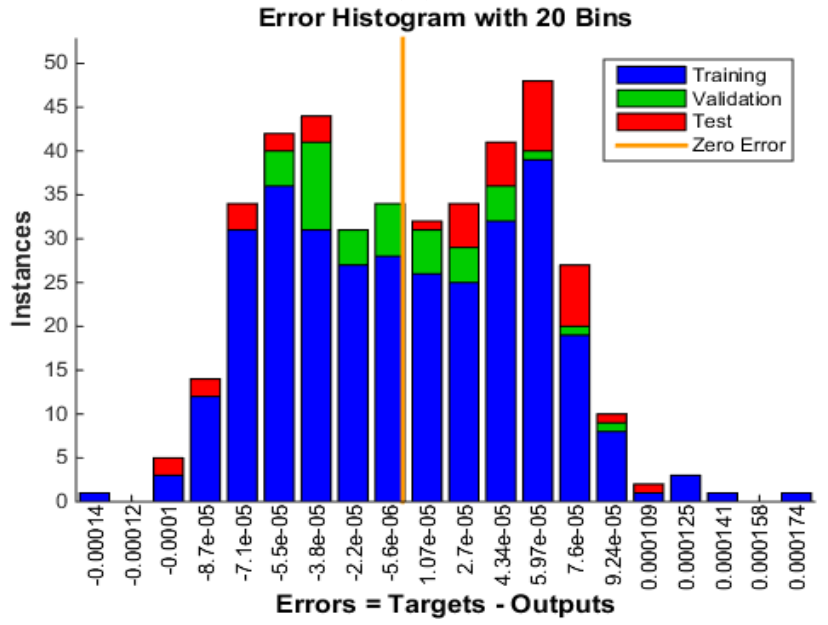

(d)
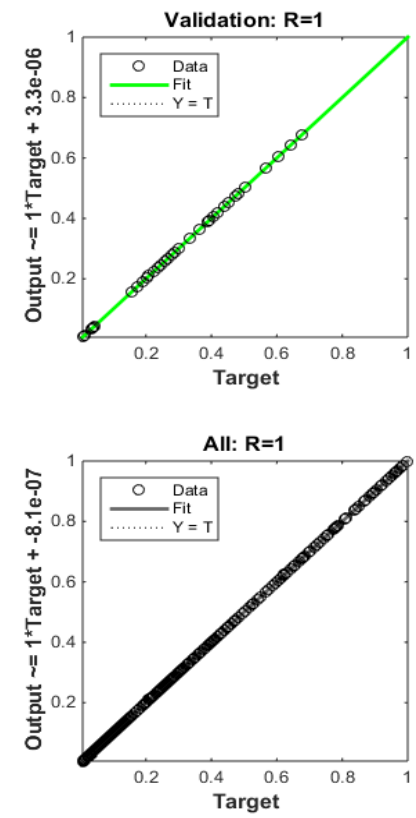

(e)

Figure 8. Graphic representation for designed LMT-ABPNN for solving cases of $M$ for $\theta(\eta)$ of MHD-SGFM. (a) MSE Result, (b) Results of the transition state, (c) Fitness of the curve, (d) Histogram for error analysis, (e) Regression. 


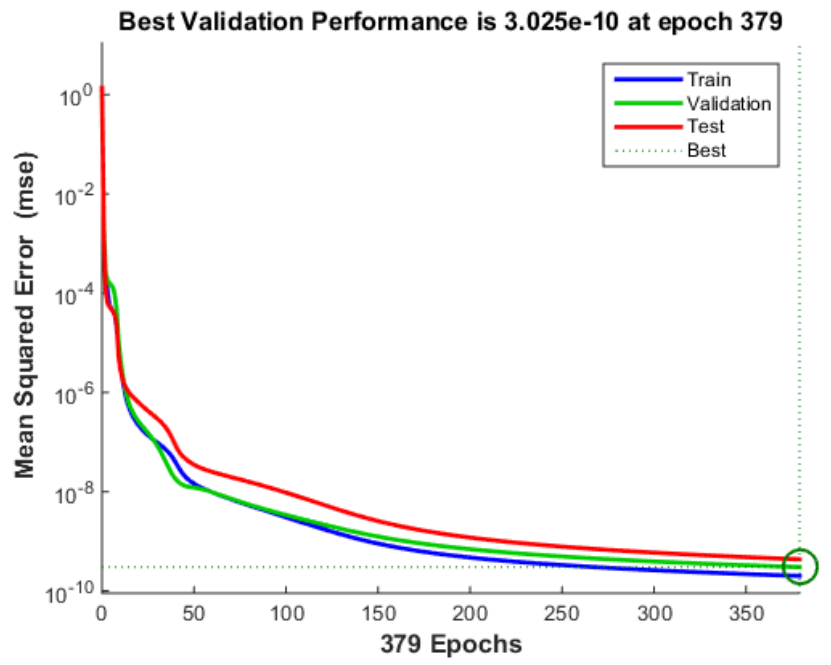

(a)

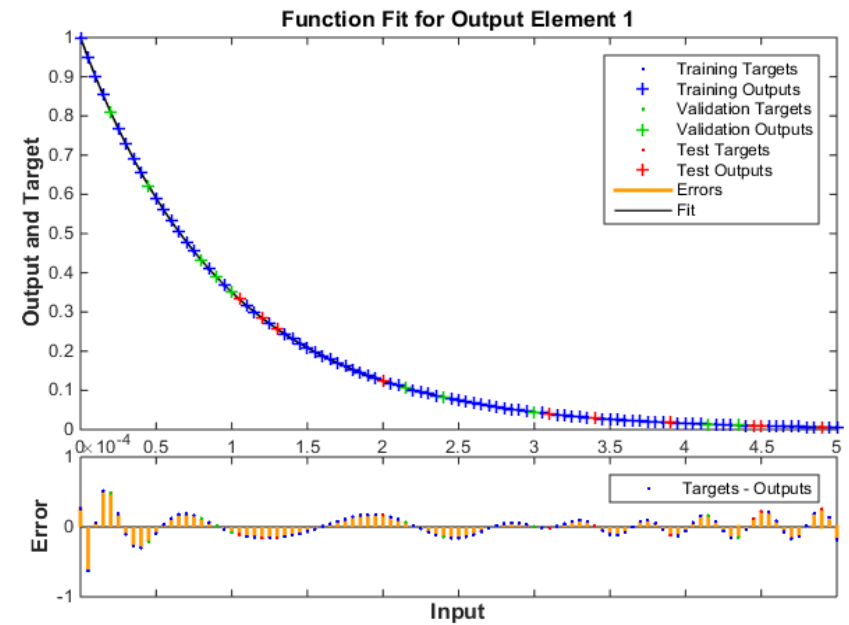

(c)
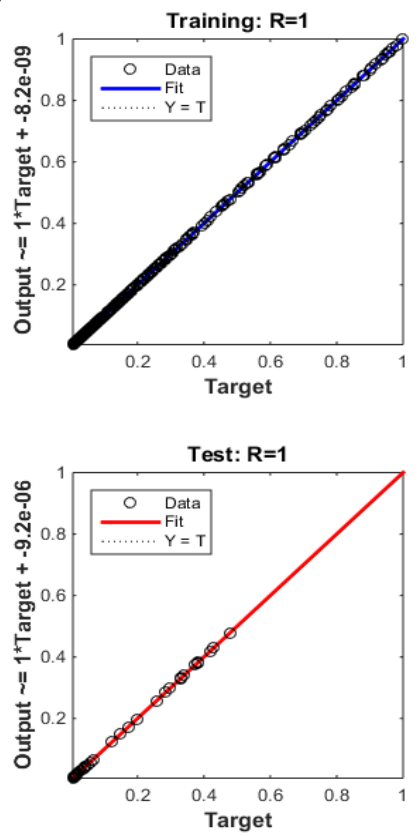

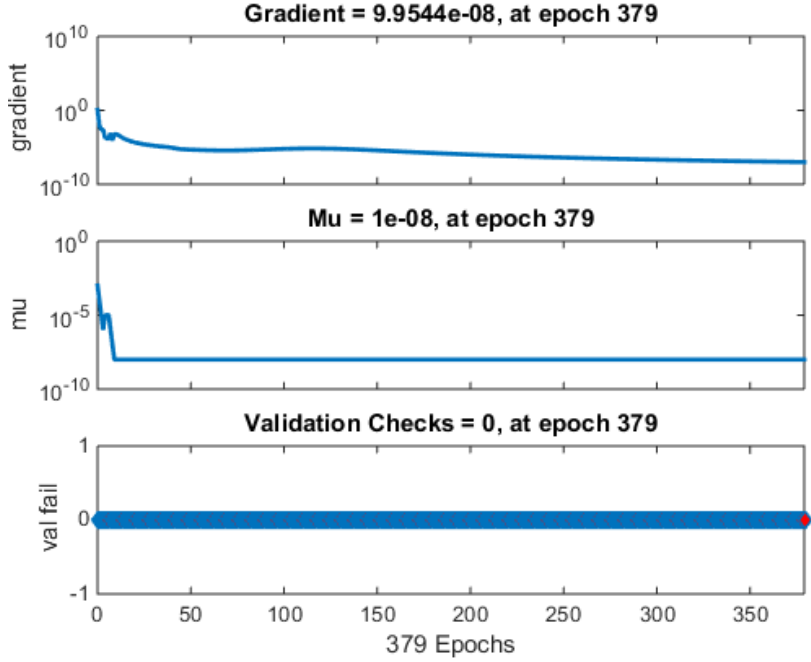

(b)

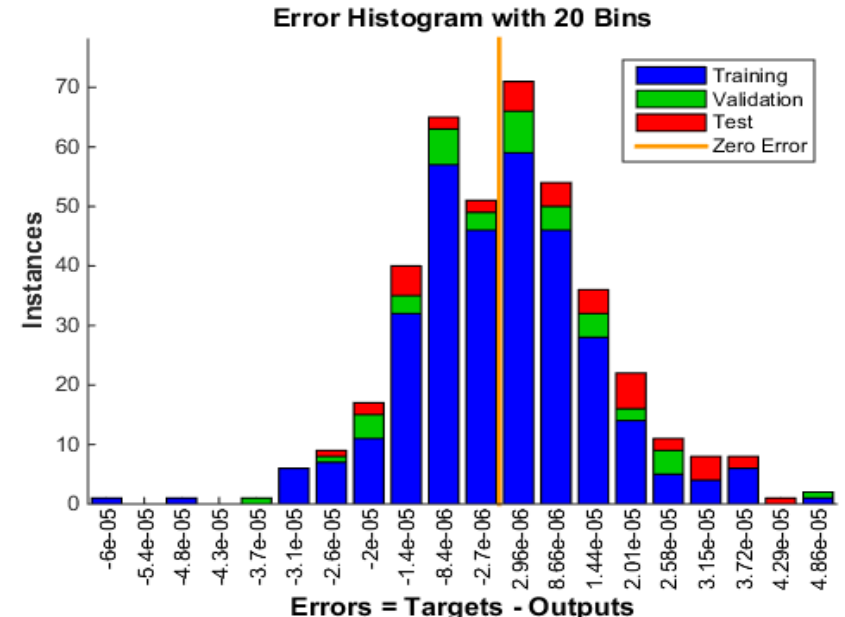

(d)
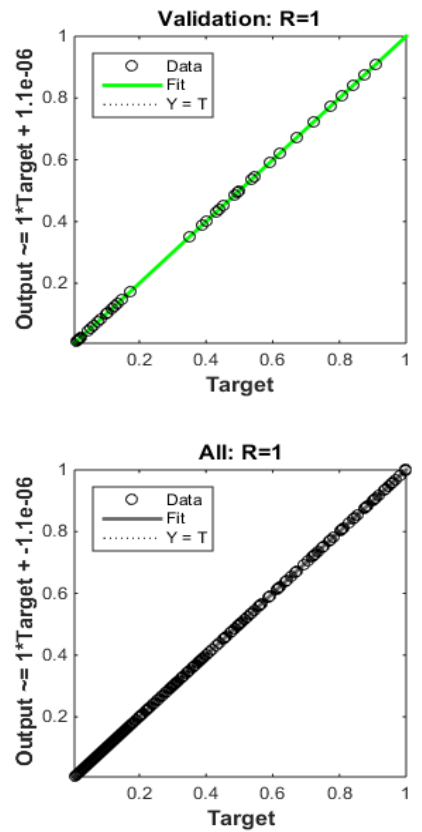

(e)

Figure 9. Graphic representation for designed LMT-ABPNN for solving cases of $M a$ for $\theta(\eta)$ of MHD-SGFM. (a) MSE Result, (b) Results of the transition state, (c) Fitness of the curve, (d) Histogram for error analysis, (e) Regression. 


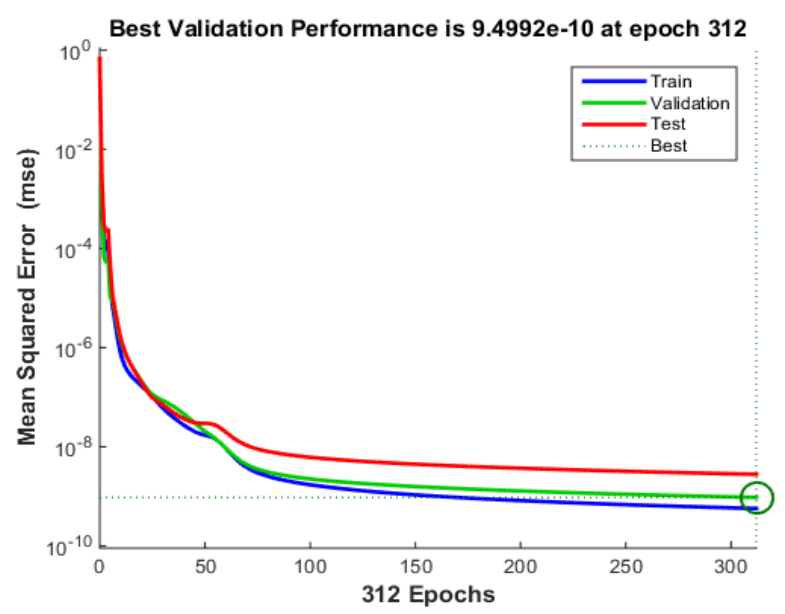

(a)

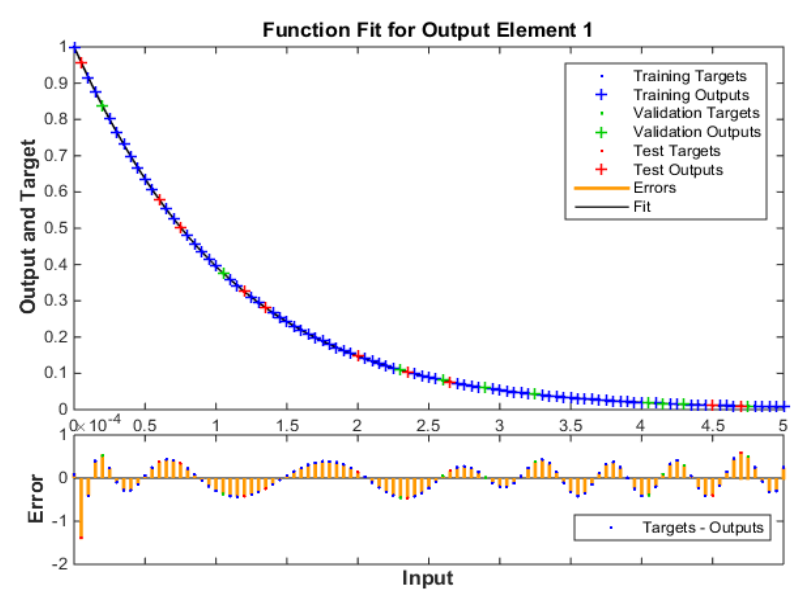

(c)
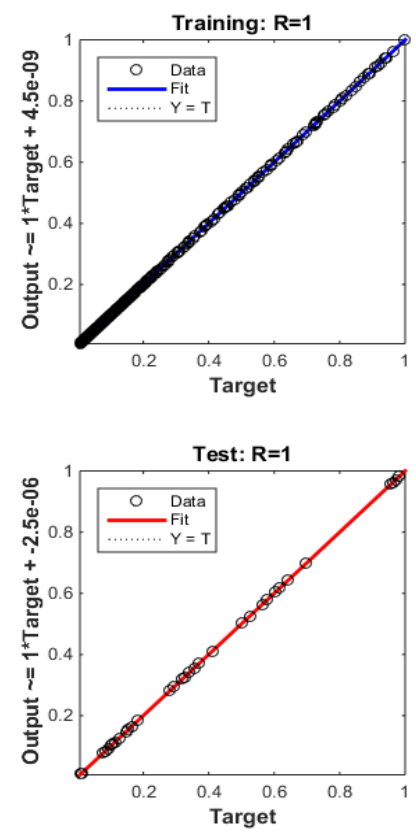
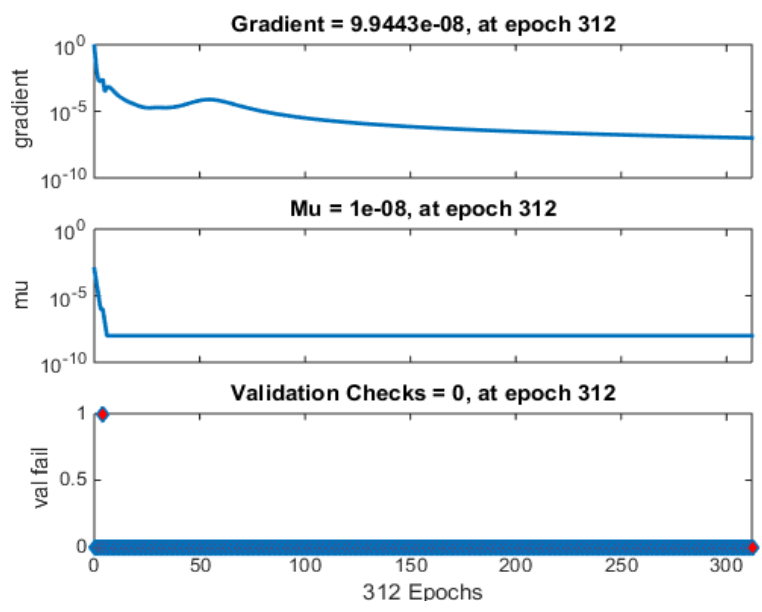

(b)

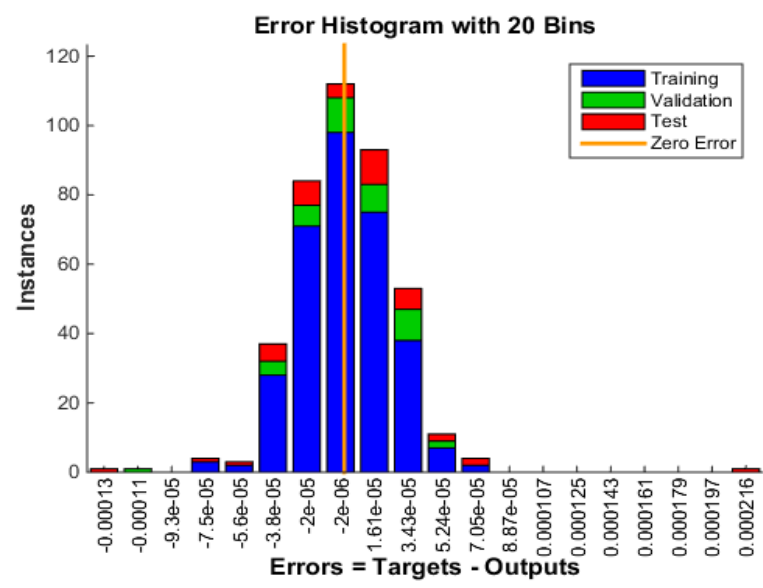

(d)
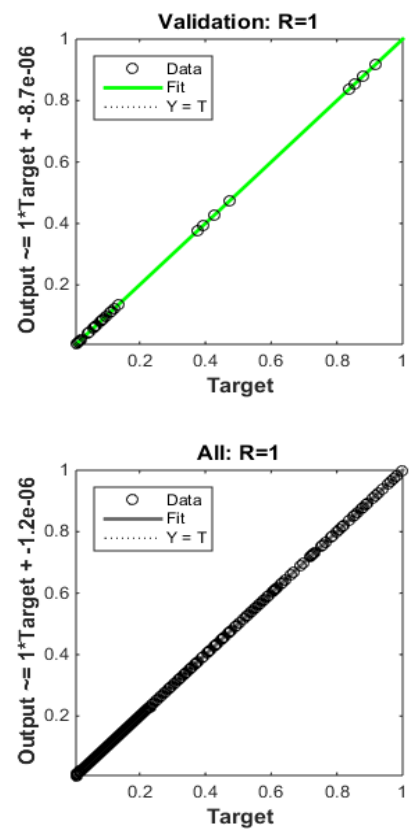

(e)

Figure 10. Graphic representation for designed LMT-ABPNN for solving cases of $E c$ for $\theta(\eta)$ of MHD-SGFM. (a) MSE Result, (b) Results of the transition state, (c) Fitness of the curve, (d) Histogram for error analysis, (e) Regression. 


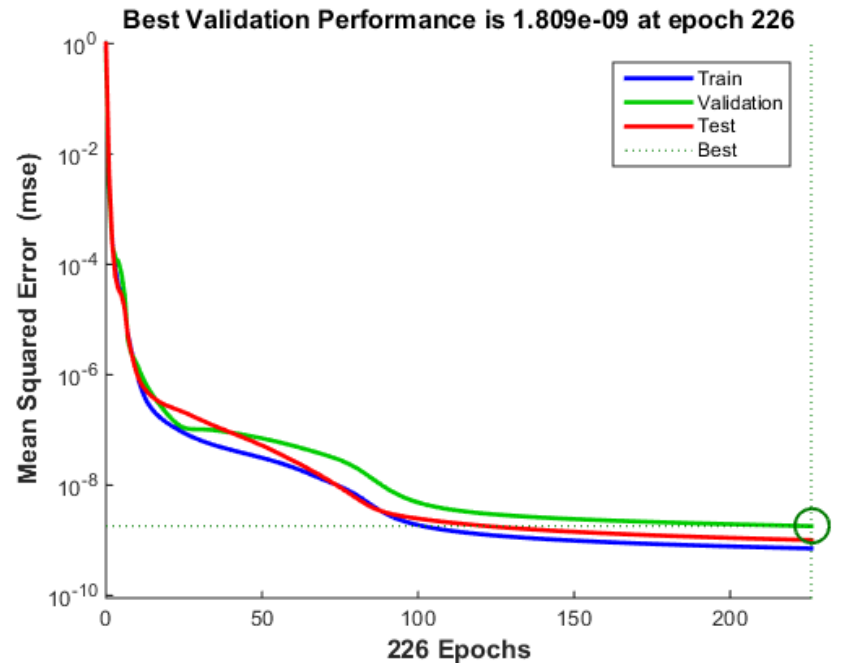

(a)

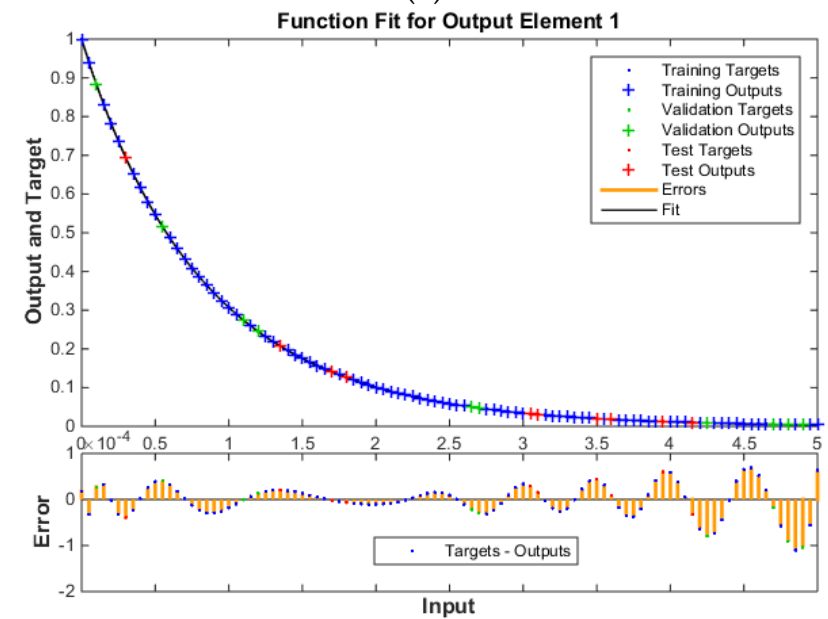

(c)
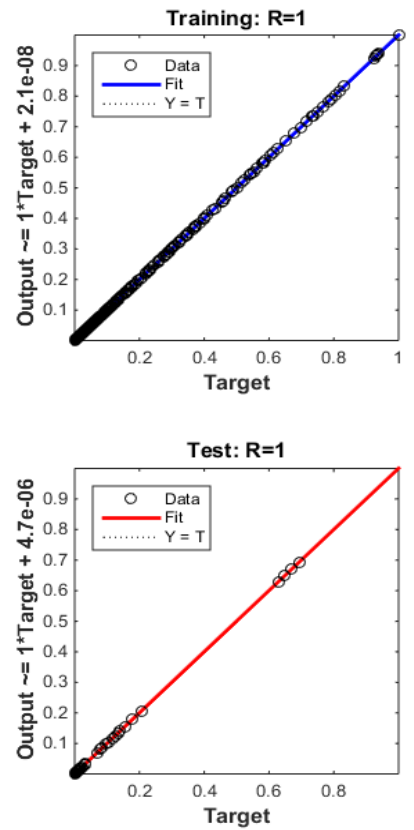

(e)

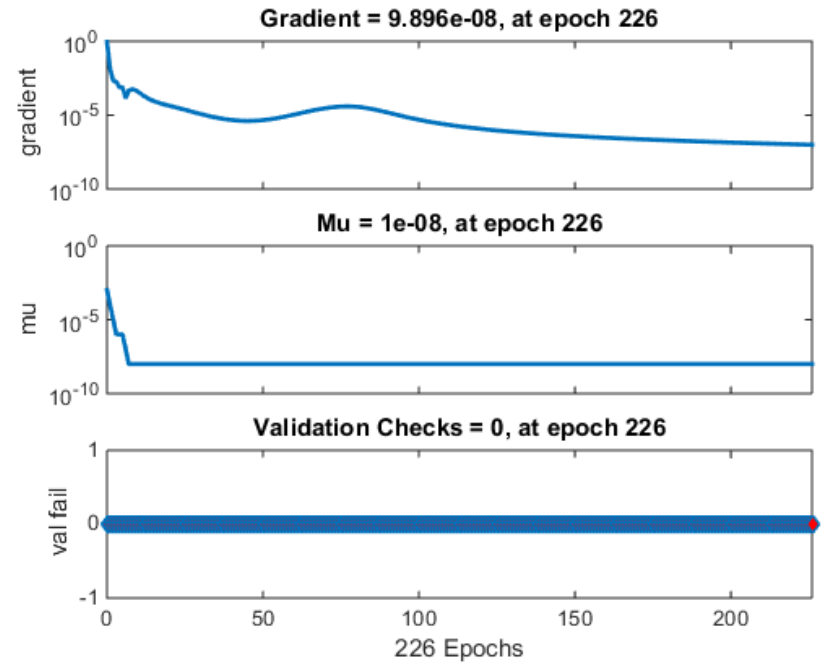

(b)

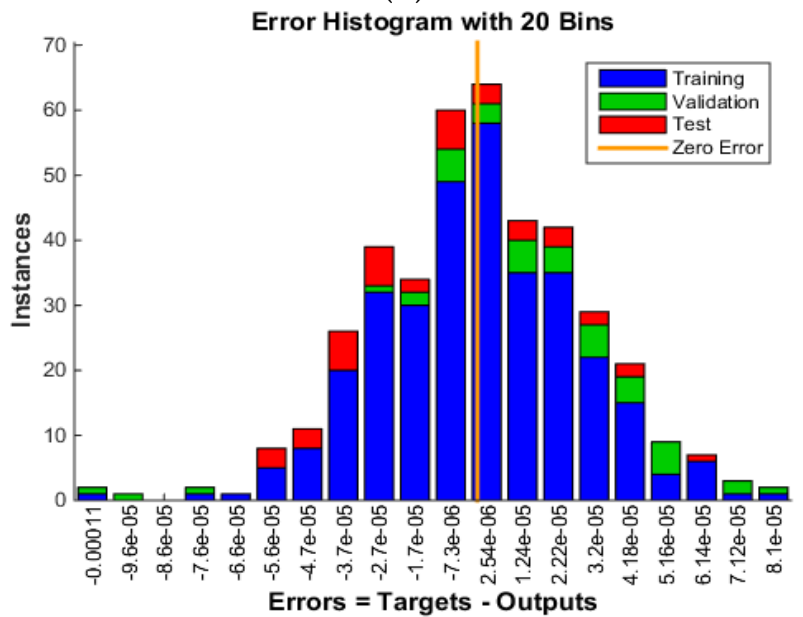

(d)
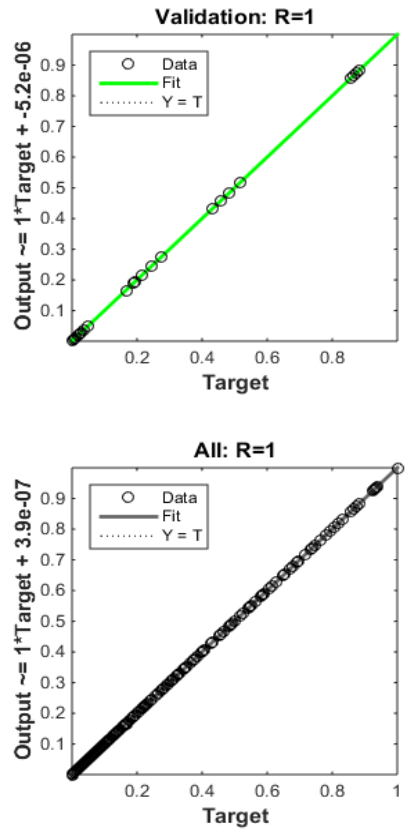

Figure 11. Graphic representation for designed LMT-ABPNN for solving cases of $M a$ for $\phi(\eta)$ of MHD-SGFM. (a) MSE Result, (b) Results of the transition state, (c) Fitness of the curve, (d) Histogram for error analysis, (e) Regression. 


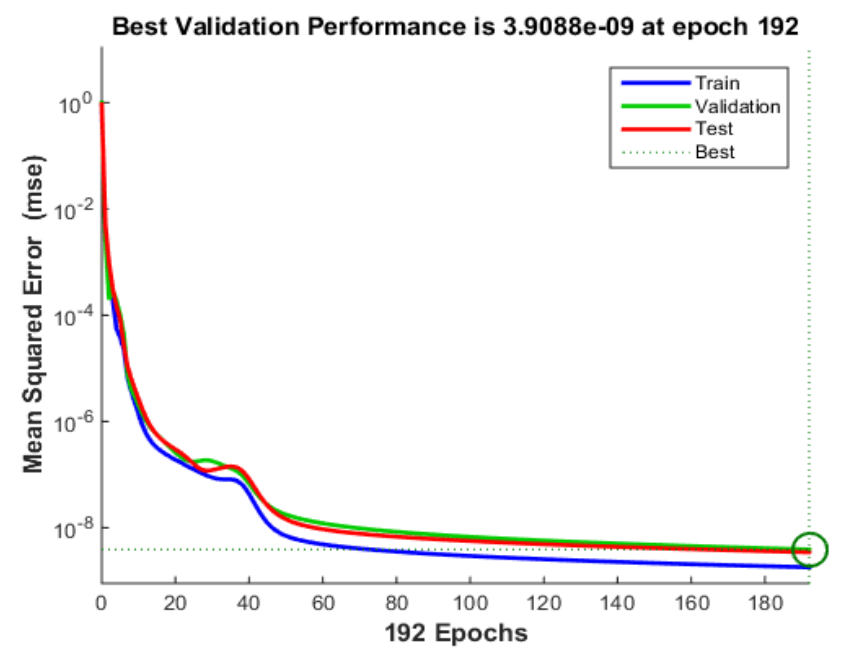

(a)

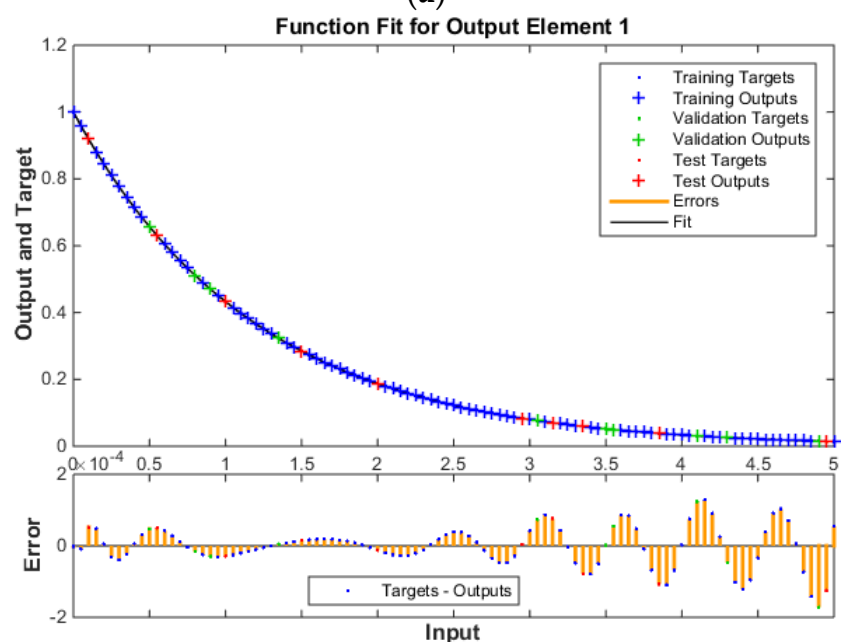

(c)
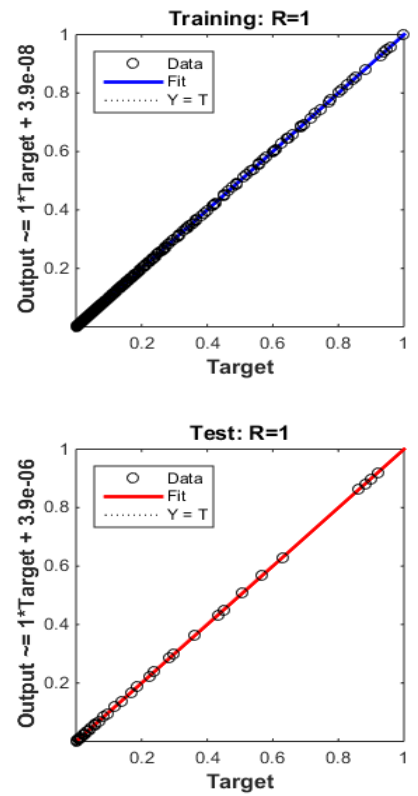

(e)

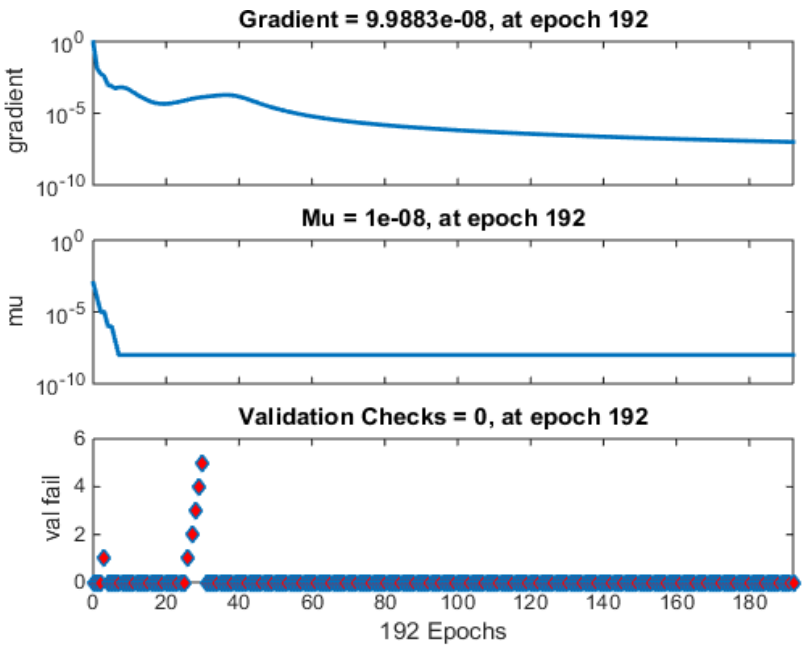

(b)

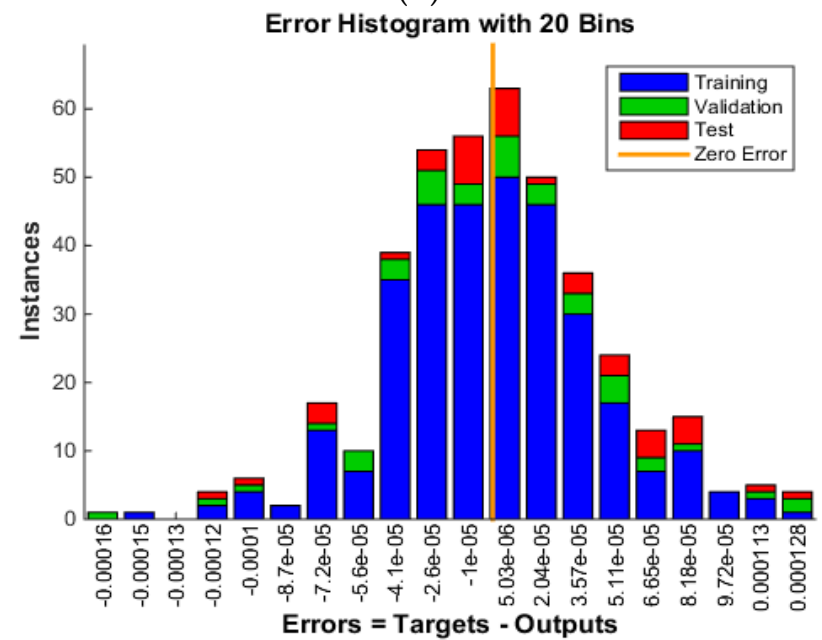

(d)
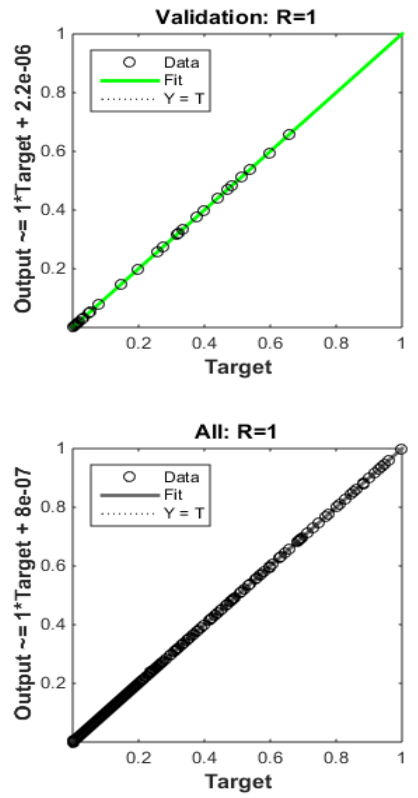

Figure 12. Graphic representation for designed LMT-ABPNN for solving cases of $S c$ for $\phi(\eta)$ of MHD-SGFM. (a) MSE Result, (b) Results of the transition state, (c) Fitness of the curve, (d) Histogram for error analysis, (e) Regression. 
The error histograms plotted in Figures 4d, 5d, 6d, 7d, 8d, 9d, 10d, 11d and 12d explain the distribution of error estimated from the zero axes after neural networks training. The error values show how the expected and target values differ. The sample numbers from the constructed data sample are represented by the instances. The greatest number of error values falls over the zero line, which shows the accuracy of the LMT-ABPNN for both scenarios and for each case, according to the error histogram analysis. Additionally, regression analysis, which is plotted in Figures 4e, 5e, 6e, 7e, 8e, 9e, 10e, 11e and 12e, is considered for training/validation/testing of the scenarios. The data prediction and forecasting were investigated in this analysis. During the computation, the regression value $R=1$ represents the close correlation between the target and output values.

\section{Impact of Profiles}

\subsection{Influence on Velocity Gradient $f^{\prime}(\eta)$}

The comparative analysis of $f^{\prime}(\eta)$ with reference solution is depicted in Figure 13 for variation of $\beta, M$, and $M a$. Figure 13a reveals behavior of $\beta$ with respect to $f^{\prime}(\eta)$. A greater value of $\beta$ results to a lower viscosity of the fluid, which minimizes the resistive force and hence raises $f^{\prime}(\eta)$. The fluctuation of $M$ via $f^{\prime}(\eta)$ is shown in Figure 13c. The disruption to the liquid particles improves when the Hartmann number is increased, and so decreases the $f^{\prime}(\eta)$. The behaviour of the $M a$ on $f^{\prime}(\eta)$ is demonstrated in Figure 13e. The velocity $f^{\prime}(\eta)$ is increased by higher estimation of $M a$. Physically, as $M a$ increases, the solutal and thermal surface tension ratios increase, improving liquid mobility. To check the accuracy criteria the absolute error (AE) analysis is plotted in Figure $13 \mathrm{~b}, \mathrm{~d}, \mathrm{f}$ for $\beta, M$, and $M a$, respectively. The AE values for $\beta$ lie between $10^{-6}$ to $10^{-3}$, for $M$ they lie between $10^{-8}$ to $10^{-4}$, while for $M a$ they lie between $10^{-7}$ to $10^{-4}$. All these AE values from reference solution satisfy the accuracy criteria.

\subsection{Influence on Temperature Gradient $\theta(\eta)$}

The comparative analysis of $\theta(\eta)$ with reference solution is depicted in Figure 14 for variation of $\beta, M, M a$, and $E c$. Figure 14 a reveals behavior of $\beta$ with respect to $\theta(\eta)$. For higher values of $\beta$ the temperature increases. Figure $14 \mathrm{c}$ depicts the variation of $M$ against $\theta(\eta)$. The temperature rises as $M$ increases. In fact, when $M$ increases, more resistive force is applied to the liquid particles, which increases collisions and, as a result, $\theta(\eta)$ rises. In Figure $14 \mathrm{e}$, the characteristics of $M a$ on $\theta(\eta)$ are highlighted. In this case, the temperature distribution is enhanced for higher estimation of $M a$. Figure $14 \mathrm{~g}$ depicts the significant effect of $E c$ on $\theta(\eta)$. Higher $E c$ approximations clearly provide more kinetic energy, which boosts temperature distribution. To check the accuracy criteria the absolute error (AE) analysis is plotted in Figure $14 \mathrm{~b}, \mathrm{~d}, \mathrm{f}, \mathrm{h}$ for $\beta, M, M a$ and $E c$, respectively. The AE values for $\beta$ lie between $10^{-7}$ to $10^{-4}$, for $M$ between $10^{-7}$ to $10^{-3}$, while for $M a$ and Ec they lie between $10^{-8}$ to $10^{-4}$. All these AE values fulfill the precision criteria.

\subsection{Influence on Concentration Field $\phi(\eta)$}

The comparative analysis of $\phi(\eta)$ with reference solution is depicted in Figure 15 for variation of $M a$ and $S c$. The behaviour of $M a$ on concentration is estimated in Figure 15a. Figure 15a reveals that $\phi(\eta)$ decays for $M a$. Surface tension is created as a result of temperature and concentration gradients, and so $\phi(\eta)$ reduced. Figure $15 \mathrm{c}$ shows the variation in concentration due to $S c$. The mass diffusivity decreases as the Schmidt number rises, consequently $\phi(\eta)$ decays. The AE analysis is depicted for $M a$ and $S c$ in Figure 15b,d. The AE values for $M a$ lies between $10^{-7}$ to $10^{-4}$, while for $S c$ lies between $10^{-7}$ to $10^{-3}$, which measures the accuracy criteria. 


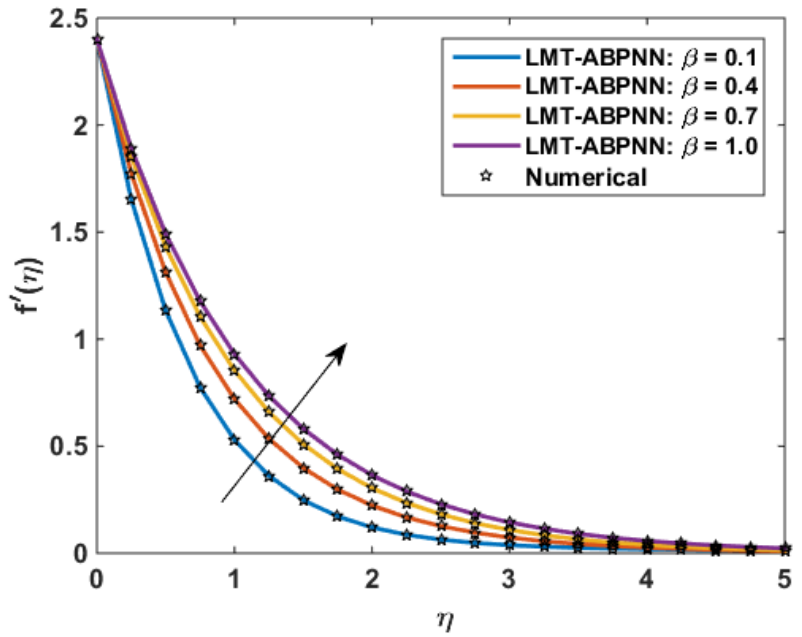

(a)

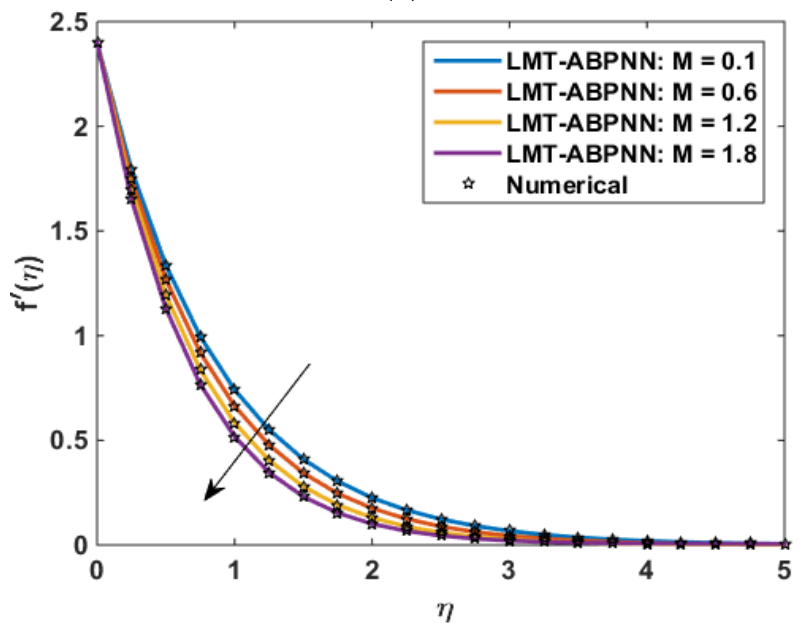

(c)

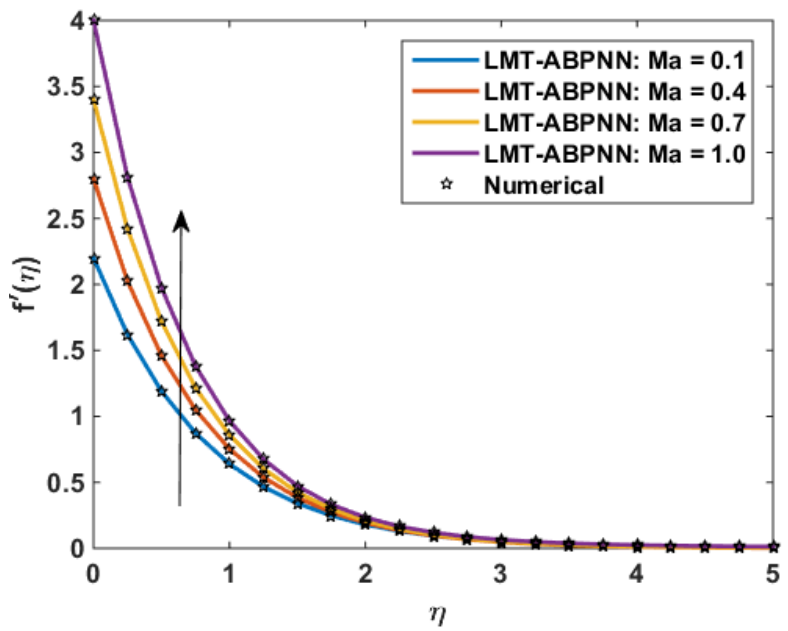

(e)

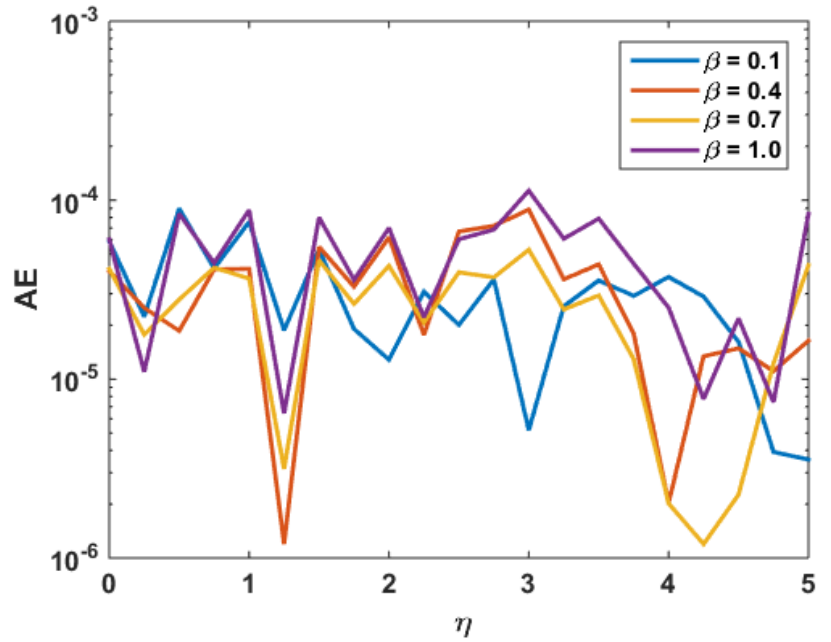

(b)

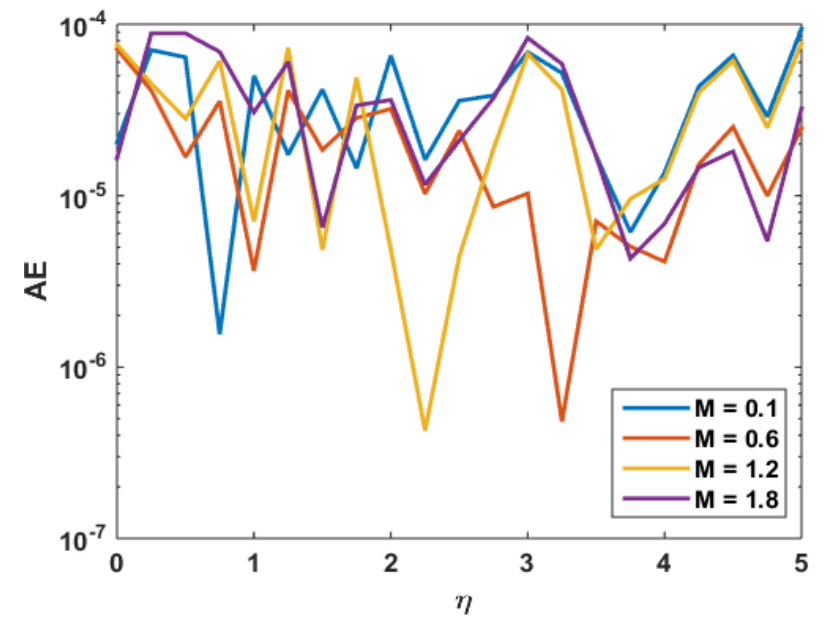

(d)

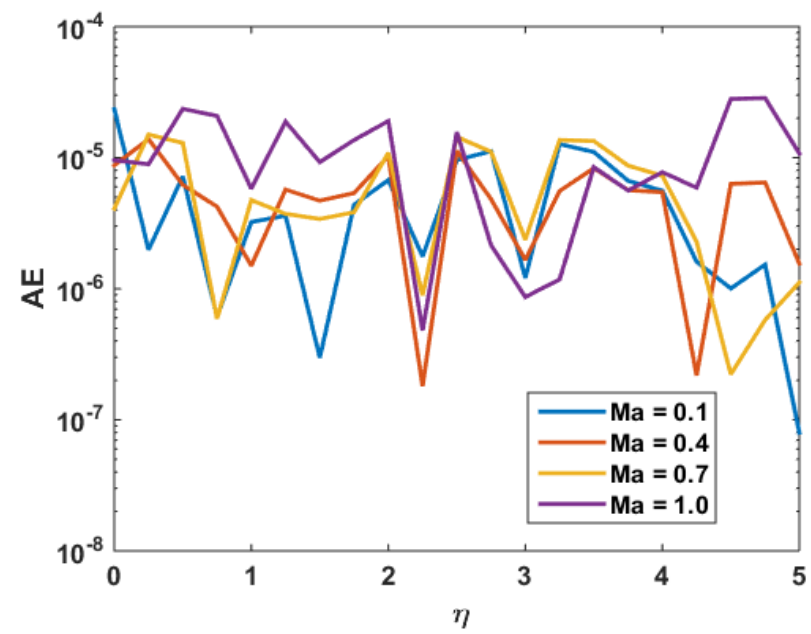

(f)

Figure 13. Assessment of LMT-ABPNN for $f^{\prime}(\eta)$ with reference dataset of MHD-SGFM. (a) For variation of $\beta$ for $\mathrm{f}^{\prime}$, (b) AE for MHD-SGFM, (c) For variation of $M$ for $\mathrm{f}^{\prime}$, (d) AE for MHD-SGFM, (e) For variation of Ma for $\mathrm{f}^{\prime}$, (f) AE for MHD-SGFM. 


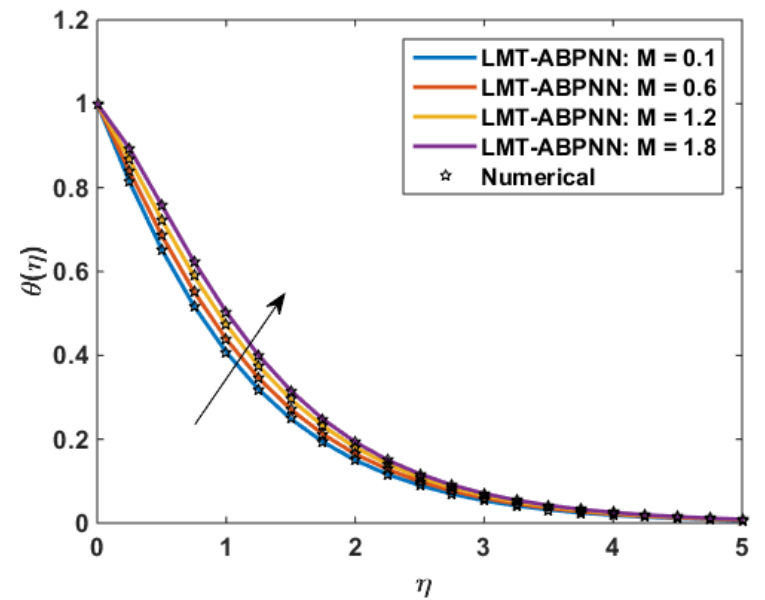

(c)

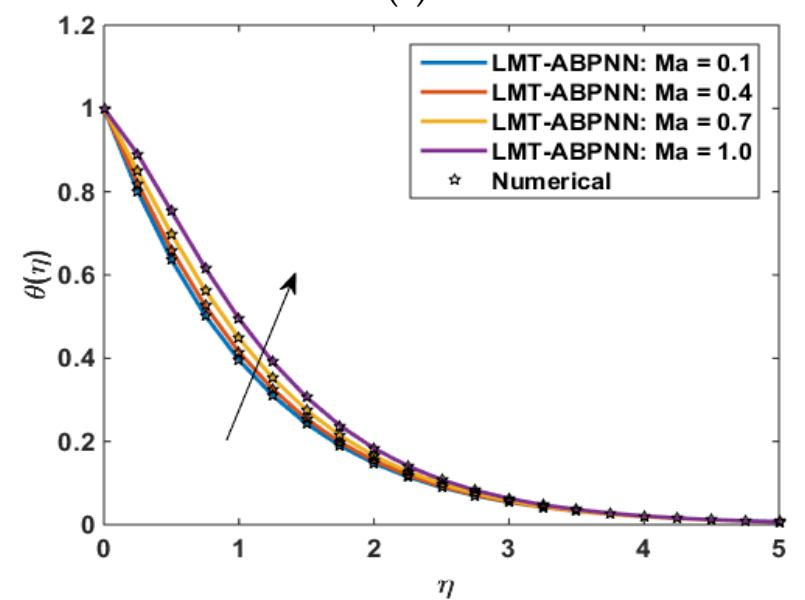

(e)

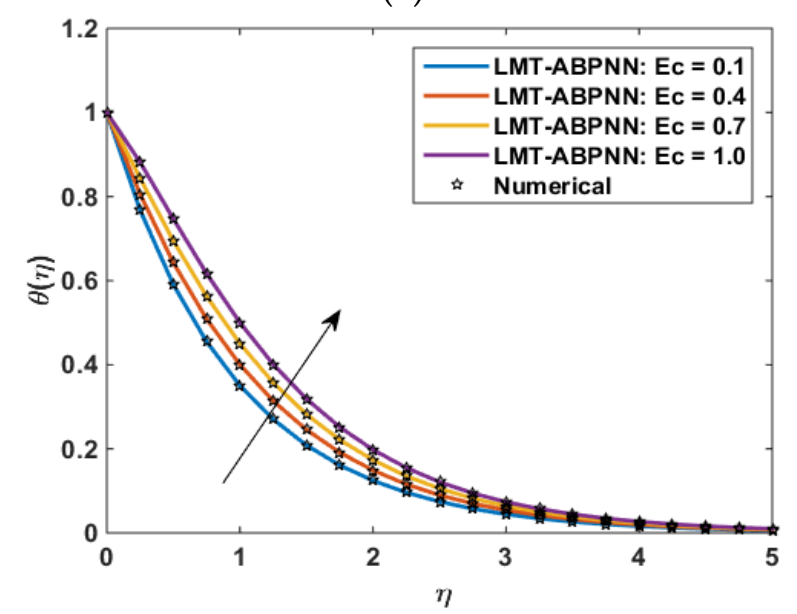

(g)

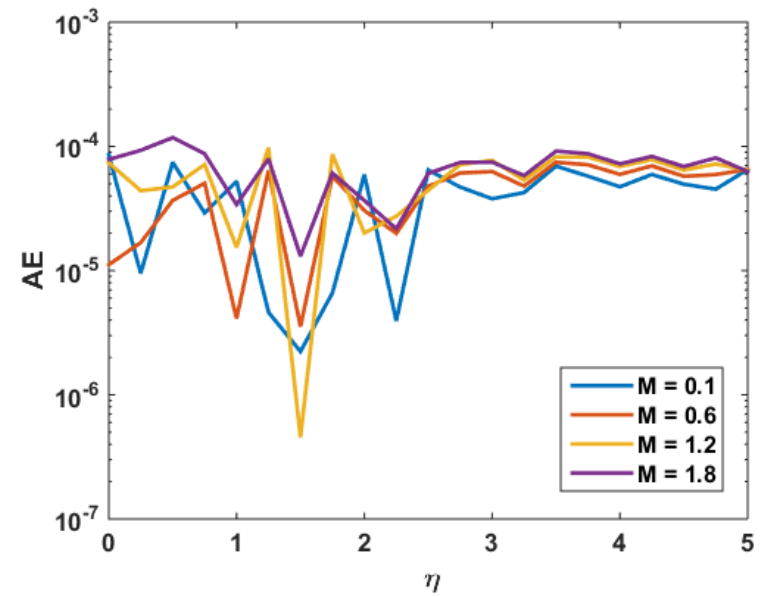

(d)

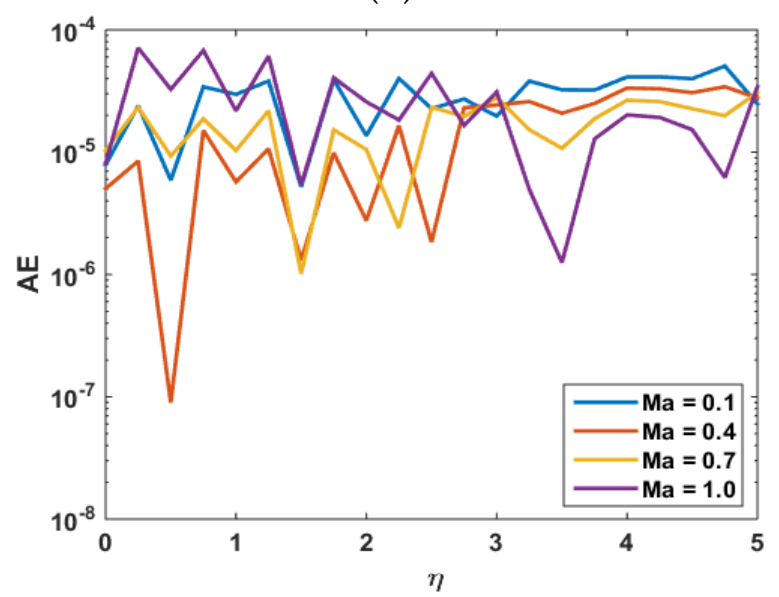

(f)

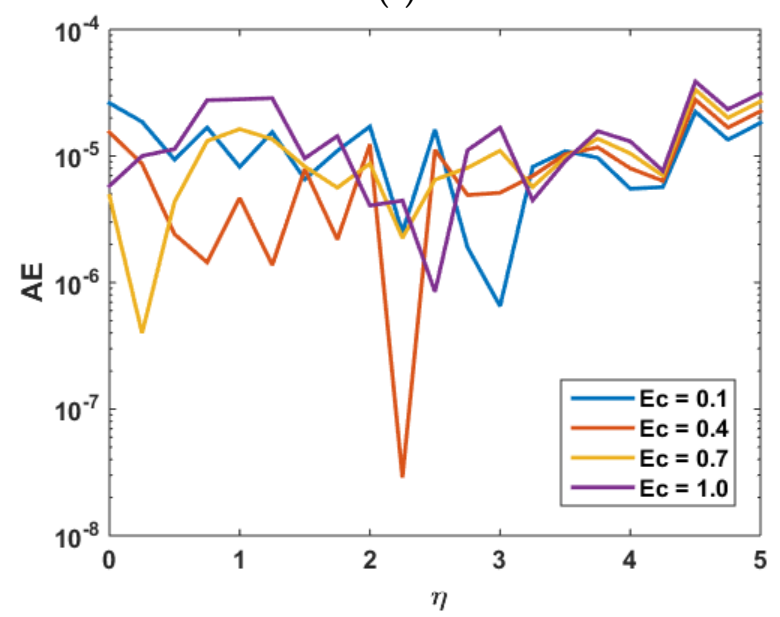

(h)

Figure 14. Assessment of LMT-ABPNN for $\theta(\eta)$ with reference dataset of MHD-SGFM. (a) For variation of $\beta$ for $\theta$, (b) AE for MHD-SGFM, (c) For variation of $M$ for $\theta$, (d) AE for MHD-SGFM, (e) For variation of Ma for $\theta$, (f) AE for MHD-SGFM, (g) For variation of Ec for $\theta$, (h) AE for MHD-SGFM. 


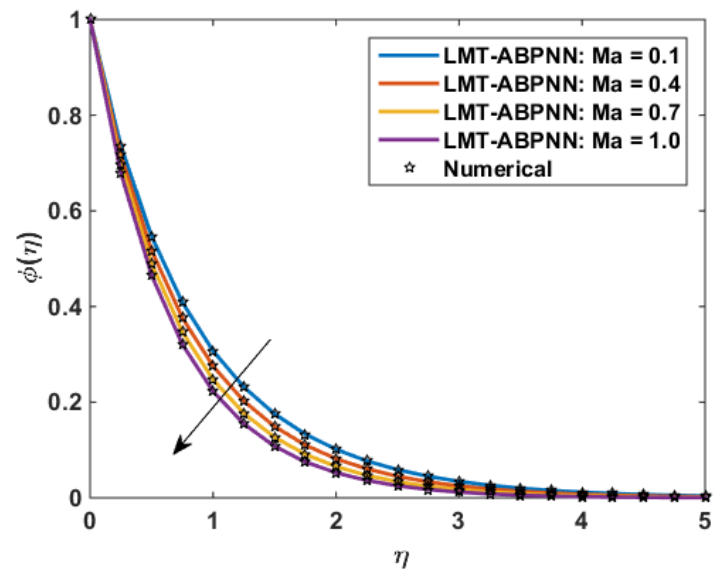

(a)

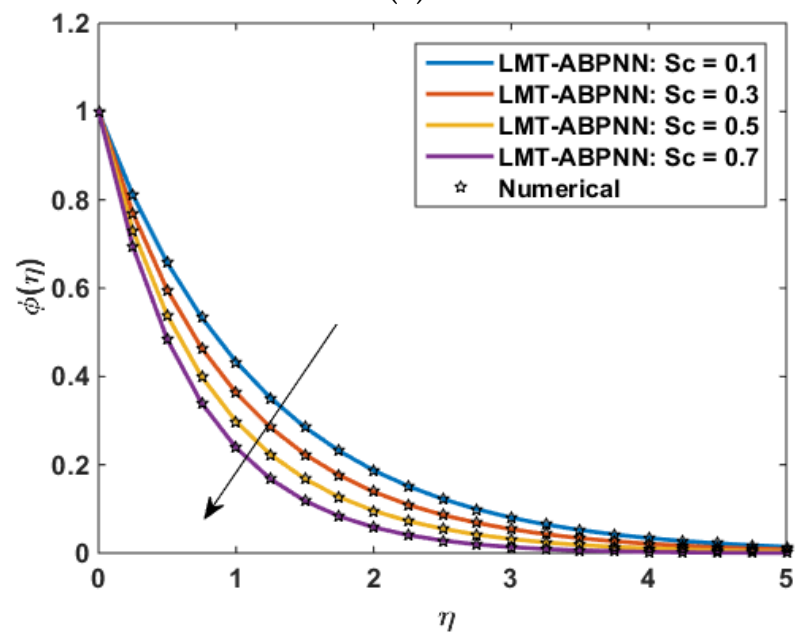

(c)

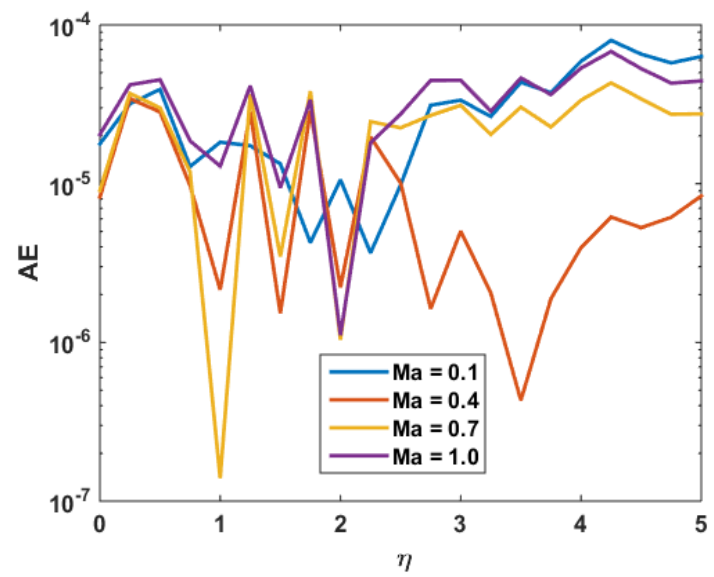

(b)

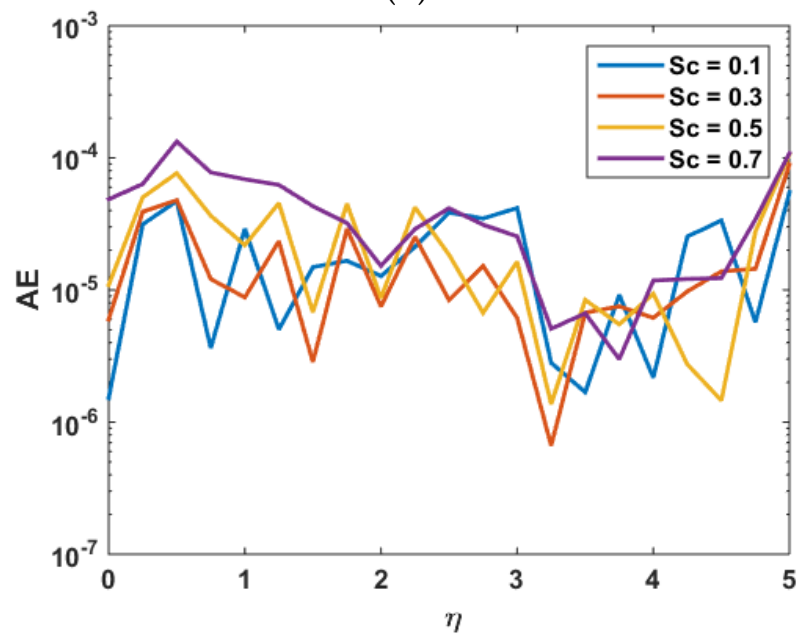

(d)

Figure 15. Assessment of LMT-ABPNN for $\phi(\eta)$ with reference dataset of MHD-SGFM. (a) For variation of $M a$ for $\phi$, (b) AE for MHD-SGFM, (c) For variation of $S c$ for $\phi$, (d) AE for MHD-SGFM.

\subsection{Entropy Generation and Bejan Number Analysis}

Figure 16 shows the effects of $\beta, M$, and $B r$ on $N_{G}$ and $B e$. Figure $16 \mathrm{a}, \mathrm{b}$ shows the effects of fluid parameter $\beta$ on $N_{G}$ and $B e$. It is noticed that the effects of $N_{G}$ and $B e$ on the fluid parameter are opposite to each other. Figure 16c,d depicts the impact of the Hartmann number $(M)$ on $N_{G}$ and $B e$. In this case, the entropy rate increases as $M$ increases. Physically, higher $M$ results in increased resistance to liquid particles, which increases the collision of molecules and, as a result, creates disorder in the thermal system. As a result, $N_{G}$ is increased. For larger $M, B e$ is reduced. Figure $16 e, f$ shows $B r$ fluctuation on $N_{G}$ and $B e$. Here, $N_{G}$ is improved for $B r$. With greater $B r$, the viscous force increases, which raises the resistive force, increasing system disorderliness, and therefore increasing $N_{G}$. The $B e$ is decreased for better estimates of $B r$. 


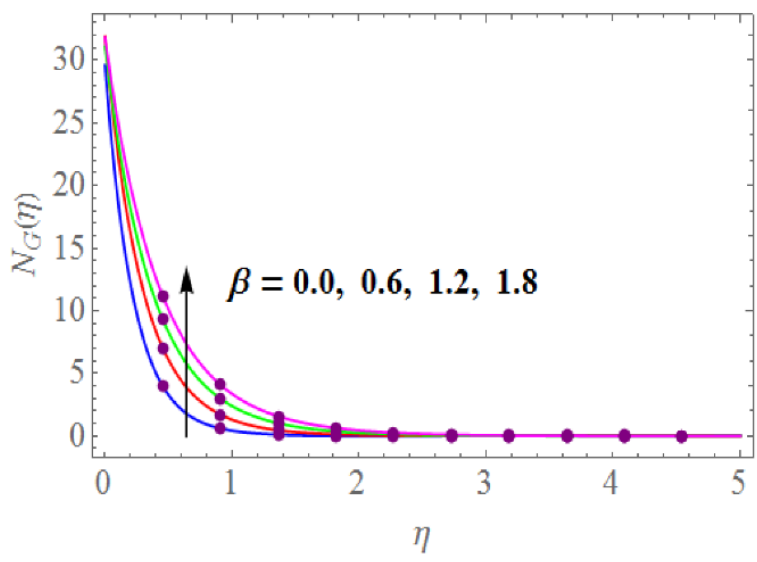

(a)

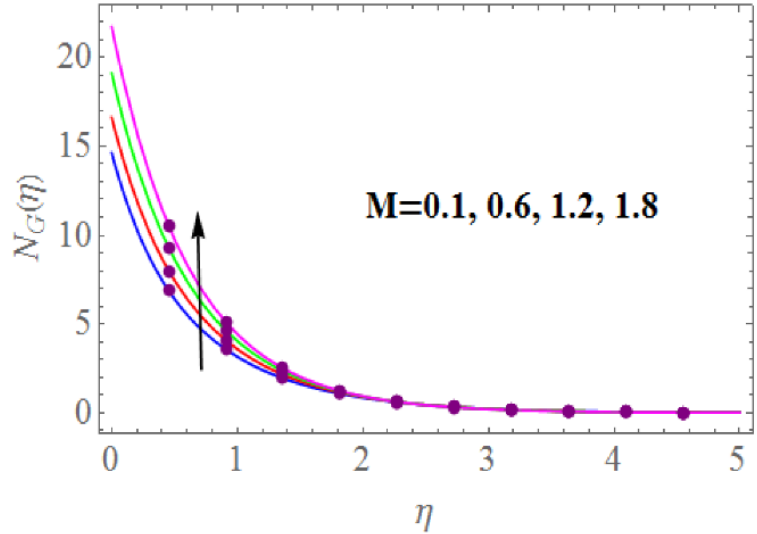

(c)

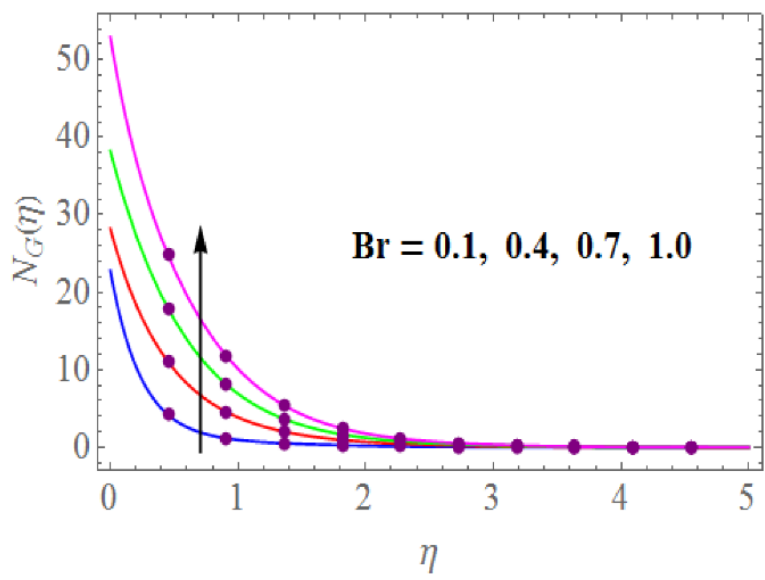

(e)

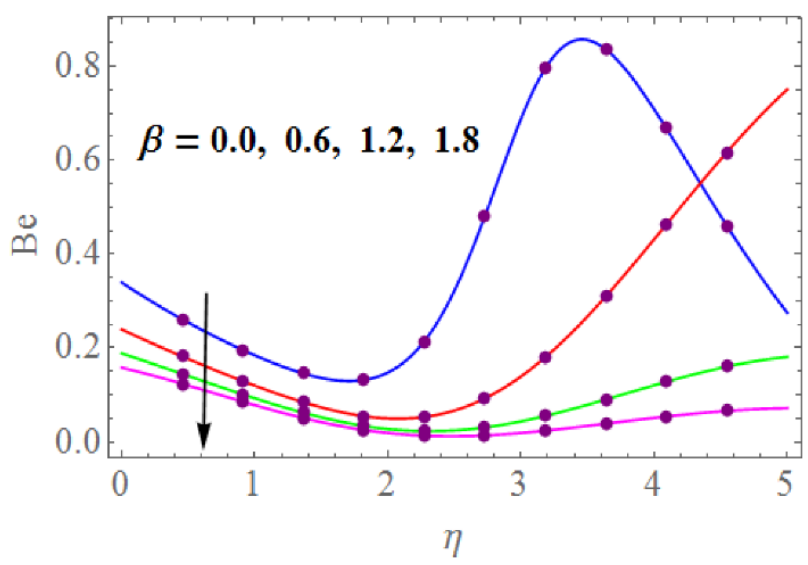

(b)

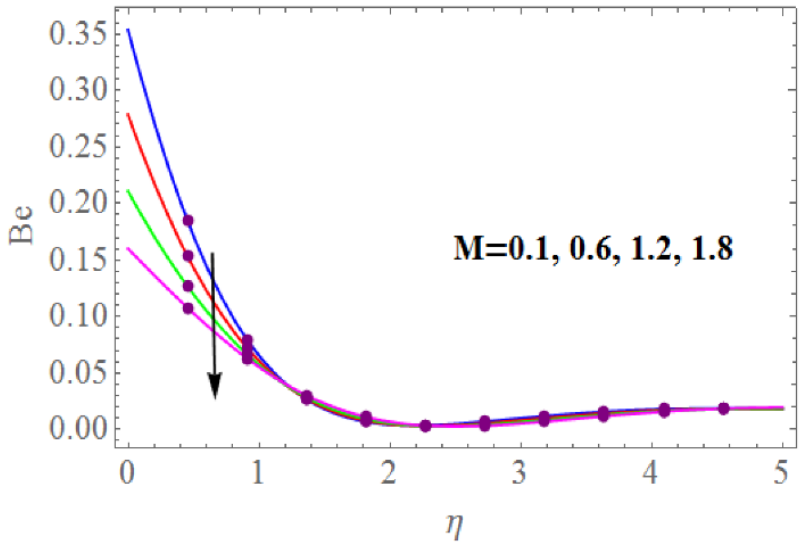

(d)

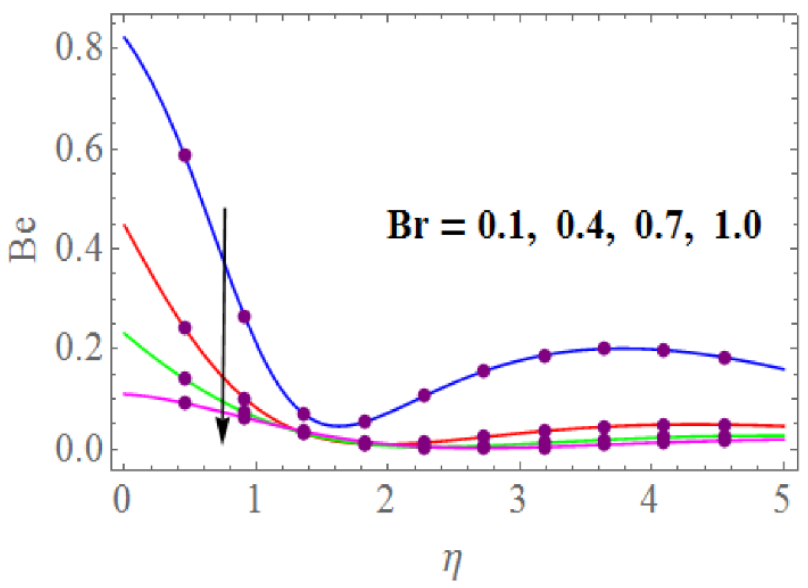

(f)

Figure 16. Entropy Generation and Bejan Number Analysis of MHD-SGFM. (a) Variation of $\beta$ for $N_{G}(\eta)$, (b) Variation of $\beta$ for $B e$, (c) Variation of $M$ for $N_{G}(\eta)$, (d) Variation of $M$ for $B e,(\mathbf{e})$ Variation of $B r$ for $N_{G}(\eta)$, (f) Variation of $B r$ for $B e$.

\section{Conclusions}

An innovative application of LMT-ABPNN has been introduced to examine the entropy generation in Marangoni convection magnetohydrodynamic second grade fluidic flow model (MHD-SGFM) with Joule heating and dissipation impact. The PDEs describing MHD-SGFM were reduced into ODEs by appropriate transformation. The dataset was determined through HAM by the variation of physical parameters for all scenarios of proposed LMT-ABPNN. The reference data samples for training/validation/testing processes 
were utilized as targets to determine the approximated solution of proposed LMT-ABPNN. The performance of LMT-ABPNN was validated by MSE based fitness, error histogram scrutiny, and regression analysis. The significant findings of this research are:

- The LMT-ABPNN's validity and verification are based on a thorough examination of accuracy assessments, histograms, and regression analysis.

- The higher the network's testing and training efficiency, the better the convergence of the produced results for the lowest $\mathrm{Mu}$ and gradient values.

- $\quad$ The larger $\beta$ and $M a$, the higher $f^{\prime}(\eta)$ while $M$ has the reverse influence on $f^{\prime}(\eta)$.

- $\quad$ For higher values of $\beta, M, M a$, and $E c, \theta(\eta)$ boosts.

- $\quad$ The concentration $\phi(\eta)$ drops as $M a$ and $S c$ grow.

- $\quad$ An augmentation is noticed for $N_{G}$ for higher estimations of $\beta, M$, and $B r$.

- $\quad$ Larger $\beta, M$, and $B r$ decays the Bejan number.

In future studies, one may work on supervised learning based LMT-ABPNN to solve the linear and nonlinear fluidic problems [50-53]. Moreover, the proposed technique is definitely helpful to exploit the different fluidic problems but other innovative heuristic unsupervised techniques can also be used.

Author Contributions: Conceptualization, M.S. and R.T.; methodology, M.A.Z.R.; software, W.J.; validation, M.I.K.; formal analysis, K.S.N.; investigation, A.R.; resources, A.-H.A.-A., I.S.Y., and E.E.M.; writing—original draft preparation, M.S. and R.T.; writing—review and editing, M.A.Z.R.; visualization, K.S.N.; supervision, M.S.; project administration, I.S.Y.; funding acquisition, A.-H.A.-A., I.S.Y. and E.E.M. All authors have read and agreed to the published version of the manuscript.

Funding: The authors express their appreciation to the Deanship of Scientific Research at King Khalid University for funding this work through the research groups program under grant number R.G.P.2/111/41. The authors extend their appreciation to the Deputyship for Research \& Innovation, Ministry of Education, in Saudi Arabia for funding this research work through the project number: (IFP-KKU-2020/9)". Emad E. Mahmoud acknowledges Taif University Researchers Supporting Project number (TURSP-2020/20), Taif University, Taif, Saudi Arabia.

Institutional Review Board Statement: Not applicable.

Informed Consent Statement: Not applicable.

Data Availability Statement: Not applicable.

Acknowledgments: The authors express their appreciation to the Deanship of Scientific Research at King Khalid University for funding this work through the research groups program under grant number R.G.P.2/111/41. The authors extend their appreciation to the Deputyship for Research \& Innovation, Ministry of Education, in Saudi Arabia for funding this research work through the project number: (IFP-KKU-2020/9)". Emad E. Mahmoud acknowledges Taif University Researchers Supporting Project number (TURSP-2020/20), Taif University, Taif, Saudi Arabia.

Conflicts of Interest: The authors declare no conflict of interest.

\section{Nomenclature}

$\begin{array}{llll}C_{\infty} & \text { Ambient concentration } & P r & \text { Prandtl number } \\ T_{\infty} & \text { Ambient Temperature of fluid } & S c & \text { Schmidt Number } \\ B e & \text { Bejan number } & T & \text { Temperature of fluid } \\ B r & \text { Brinkman Number } & k & \text { Thermal Conductivity } \\ x, y & \text { Cartesian co-ordinates } & u, v & \text { Velocity components } \\ C & \text { Concentration } & \text { Greek } & \text { Symbol } \\ A & \text { Dimensionless Parameter } & \rho & \text { Density } \\ E c & \text { Eckert number } & \mu & \text { Dynamic viscosity } \\ N_{G} & \text { Entropy optimization rate } & \sigma_{1} & \text { Electric conductivity } \\ L_{1} & \text { Reference length } & \sigma_{0}, \gamma_{T}, \gamma_{C} & \text { Positive constant } \\ B_{0} & \text { Magnetic strength } & \beta & \text { SG Fluid parameter } \\ M a & \text { Marangoni ratio parameter } & \alpha_{1} & \text { SG liquid parameter } \\ D_{B} & \text { Mass Diffusivity } & c_{p} & \text { Specific Heat }\end{array}$




\section{References}

1. Pearson, J.R.A. On convection cells induced by surface tension. J. Fluid Mech. 1958, 4, 489-500. [CrossRef]

2. Scriven, L.E.; Sternling, C.V. The marangoni effects. Nature 1960, 187, 186-188. [CrossRef]

3. Qayyum, S. Dynamics of marangoni convection in hybrid nanofluid flow submerged in ethylene glycol and water base fluids. Int. Commun. Heat Mass Transf. 2020, 119, 104962. [CrossRef]

4. Jawad, M.; Saeed, A.; Kumam, P.; Shah, Z.; Khan, A. Analysis of boundary layer MHD darcy-forchheimer radiative nanofluid flow with soret and dufour effects by means of marangoni convection. Case Stud. Therm. Eng. 2021, 23, 100792. [CrossRef]

5. Gul, T.; Ullah, M.Z.; Alzahrani, A.K.; Zaheer, Z.; Amiri, I.S. MHD Thin film flow of kerosene oil based CNTs nanofluid under the influence of marangoni convection. Phys. Scr. 2020, 95, 015702. [CrossRef]

6. Gul, T.; Akbar, R.; Zaheer, Z.; Amiri, I.S. The impact of the Marangoni convection and magnetic field versus blood-based carbon nanotube nanofluids. Proc. Inst. Mech. Eng. Part N J. Nanomater. Nanoeng. Nanosyst. 2020, 234, 37-46. [CrossRef]

7. Rasool, G.; Shafiq, A.; Khalique, C.M. Marangoni forced convective casson type nanofluid flow in the presence of lorentz force generated by riga plate. Discret. Contin. Dyn. Syst. S 2021, 14, 2517. [CrossRef]

8. Rasool, G.; Zhang, T.; Chamkha, A.J.; Shafiq, A.; Tlili, I.; Shahzadi, G. Entropy generation and consequences of binary chemical reaction on MHD Darcy-Forchheimer Williamson nanofluid flow over non-linearly stretching surface. Entropy 2020, $22,18$. [CrossRef] [PubMed]

9. Modather, M.; Chamkha, A. An Analytical study of MHD heat and mass transfer oscillatory flow of a micropolar fluid over a vertical permeable plate in a porous medium. Turk. J. Eng. Environ. Sci. 2010, 33, 245-258.

10. Krishna, M.V.; Chamkha, A.J. Hall and ion slip effects on MHD rotating boundary layer flow of nanofluid past an infinite vertical plate embedded in a porous medium. Results Phys. 2019, 15, 102652. [CrossRef]

11. Chamkha, A.J.; Issa, C.; Khanafer, K. Natural convection from an inclined plate embedded in a variable porosity porous medium due to solar radiation. Int. J. Therm. Sci. 2002, 41, 73-81. [CrossRef]

12. Chamkha, A.J. Non-darcy fully developed mixed convection in a porous medium channel with heat generation/absorption and hydromagnetic effects. Numer. Heat Transf. Part A Appl. 1997, 32, 653-675. [CrossRef]

13. Takhar, H.S.; Chamkha, A.J.; Nath, G. MHD Flow over a moving plate in a rotating fluid with magnetic field, hall currents and free stream velocity. Int. J. Eng. Sci. 2002, 40, 1511-1527. [CrossRef]

14. Takhar, H.S.; Chamkha, A.J.; Nath, G. Unsteady flow and heat transfer on a semi-infinite flat plate with an aligned magnetic field. Int. J. Eng. Sci. 1999, 37, 1723-1736. [CrossRef]

15. Chamkha, A.J. Hydromagnetic three-dimensional free convection on a vertical stretching surface with heat generation or absorption. Int. J. Heat Fluid Flow 1999, 20, 84-92. [CrossRef]

16. Ali, B.; Shafiq, A.; Siddique, I.; Al-Mdallal, Q.; Jarad, F. Significance of suction/injection, gravity modulation, thermal radiation, and magnetohydrodynamic on dynamics of micropolar fluid subject to an inclined sheet via finite element approach. Case Stud. Therm. Eng. 2021, 28, 101537. [CrossRef]

17. Ali, B.; Thumma, T.; Habib, D.; Riaz, S. Finite element analysis on transient MHD 3D rotating flow of Maxwell and tangent hyperbolic nanofluid past a bidirectional stretching sheet with Cattaneo Christov heat flux model. Therm. Sci. Eng. Prog. 2021, 101089. (In press) [CrossRef]

18. Ali, B.; Siddique, I.; Ahmadian, A.; Senu, N.; Ali, L.; Haider, A. Significance of Lorentz and Coriolis forces on dynamics of water based silver tiny particles via finite element simulation. Ain Shams Eng. J. 2021, in press. [CrossRef]

19. Li, Y.X.; Qayyum, S.; Khan, M.I.; Elmasry, Y.; Chu, Y.M. Motion of hybrid nanofluid $\left(\mathrm{MnZnFe}_{2} \mathrm{O}_{4}-\mathrm{NiZnFe}_{2} \mathrm{O}_{4}-\mathrm{H}_{2} \mathrm{O}\right) \mathrm{with}$ homogeneous-heterogeneous reaction: Marangoni convection. Math. Comput. Simul. 2021, 190, 1379-1391. [CrossRef]

20. Khan, M.I.; Qayyum, S.; Shah, F.; Kumar, R.N.; Gowda, R.P.; Prasannakumara, B.C.; Chu, Y.M.; Kadry, S. Marangoni convective flow of hybrid nanofluid ( $\mathrm{MnZnFe}_{2} \mathrm{O}_{4}-\mathrm{NiZnFe}_{2} \mathrm{O}_{4}-\mathrm{H}_{2} \mathrm{O}$ ) with Darcy Forchheimer medium. Ain Shams Eng. J. 2021, 12, $3931-3938$. [CrossRef]

21. Khan, M.I.; Qayyum, S.; Chu, Y.M.; Khan, N.B.; Kadry, S. Transportation of marangoni convection and irregular heat source in entropy optimized dissipative flow. Int. Commun. Heat Mass Transf. 2021, 120, 105031. [CrossRef]

22. Malaikah, H.; Ijaz Khan, M. Dynamics of Marangoni convection in radiative flow of power-law fluid with entropy optimization. Int. J. Mod. Phys. B 2021, 35, 2150280. [CrossRef]

23. Ahmed, S.E.; Oztop, H.F.; Elshehabey, H.M. Thermosolutal Marangoni convection of bingham non-Newtonian fluids within inclined lid-driven enclosures full of porous media. Heat Transf. 2021, 50, 7898-7917. [CrossRef]

24. Khan, M.I.; Qayyum, S.; Chu, Y.M.; Kadry, S. Numerical simulation and modeling of entropy generation in Marangoni convective flow of nanofluid with activation energy. Numer. Methods Partial. Differ. Equ. 2020, in press. [CrossRef]

25. Khan, S.A.; Hayat, T.; Alsaedi, A.; Zai, Q.Z. Irreversibility analysis in Marangoni forced convection flow of second grade fluid. J. Phys. Commun. 2020, 4, 085013. [CrossRef]

26. Abiodun, O.I.; Jantan, A.; Omolara, A.E.; Dada, K.V.; Mohamed, N.A.; Arshad, H. State-of-the-art in artificial neural network applications: A survey. Heliyon 2018, 4, e00938. [CrossRef]

27. Bui, D.K.; Nguyen, T.N.; Ngo, T.D.; Nguyen-Xuan, H. An artificial neural network (ANN) expert system enhanced with the electromagnetism-based firefly algorithm (EFA) for predicting the energy consumption in buildings. Energy 2020, 190, 116370. [CrossRef] 
28. Zhao, Z.; Lou, Y.; Chen, Y.; Lin, H.; Li, R.; Yu, G. Prediction of interfacial interactions related with membrane fouling in a membrane bioreactor based on radial basis function artificial neural network (ANN). Bioresour. Technol. 2019, 282, 262-268. [CrossRef] [PubMed]

29. Moayedi, H.; Mosallanezhad, M.; Rashid, A.S.A.; Jusoh, W.A.W.; Muazu, M.A. A systematic review and meta-analysis of artificial neural network application in geotechnical engineering: Theory and applications. Neural Comput. Appl. 2020, 32, 495-518. [CrossRef]

30. Bukhari, A.H.; Sulaiman, M.; Islam, S.; Shoaib, M.; Kumam, P.; Raja, M.A.Z. Neuro-fuzzy modeling and prediction of summer precipitation with application to different meteorological stations. Alex. Eng. J. 2020, 59, 101-116. [CrossRef]

31. Xia, M.; Zheng, X.; Imran, M.; Shoaib, M. Data-driven prognosis method using hybrid deep recurrent neural network. Appl. Soft Comput. 2020, 93, 106351. [CrossRef]

32. Khan, R.A.; Ullah, H.; Raja, M.A.Z.; Khan, M.A.R.; Islam, S.; Shoaib, M. Heat transfer between two porous parallel plates of steady nano fludis with Brownian and thermophoretic effects: A new stochastic numerical approach. Int. Commun. Heat Mass Transf. 2021, 126, 105436. [CrossRef]

33. Aljohani, J.L.; Alaidarous, E.S.; Raja, M.A.Z.; Shoaib, M.; Alhothuali, M.S. Intelligent computing through neural networks for numerical treatment of non-Newtonian wire coating analysis model. Sci. Rep. 2021, 11, 9072. [CrossRef]

34. Shah, Z.; Raja, M.A.Z.; Chu, Y.M.; Khan, W.A.; Abbas, S.Z.; Shoaib, M.; Irfan, M. Computational intelligence of LevenbergMarquardt backpropagation neural networks to study the dynamics of expanding/contracting cylinder for cross magnetonanofluid flow model. Phys. Scr. 2021, 96, 055219. [CrossRef]

35. Shoaib, M.; Raja, M.A.Z.; Jamshed, W.; Nisar, K.S.; Khan, I.; Farhat, I. Intelligent computing Levenberg Marquardt approach for entropy optimized single-phase comparative study of second grade nanofluidic system. Int. Commun. Heat Mass Transf. 2021, 127, 105544. [CrossRef]

36. Raja, M.A.Z.; Shoaib, M.; Tabassum, R.; Khan, M.I.; Gowda, R.P.; Prasannakumara, B.C.; Malik, M.Y.; Xia, W.F. Intelligent computing for the dynamics of entropy optimized nanofluidic system under impacts of MHD along thick surface. Int. J. Mod. Phys. B 2021, 35, 2150269. [CrossRef]

37. Raja, M.A.Z.; Shoaib, M.; Khan, Z.; Zuhra, S.; Saleel, C.A.; Nisar, K.S.; Islam, S.; Khan, I. Supervised neural networks learning algorithm for three dimensional hybrid nanofluid flow with radiative heat and mass fluxes. Ain Shams Eng. J. 2021, in press. [CrossRef]

38. Ilyas, H.; Ahmad, I.; Raja, M.A.Z.; Tahir, M.B.; Shoaib, M. Neuro-intelligent mappings of hybrid hydro-nanofluid $\mathrm{Al}_{2} \mathrm{O}_{3}-\mathrm{Cu}-\mathrm{H}_{2} \mathrm{O}$ model in porous medium over rotating disk with viscous dissolution and JOULE heating. Int. J. Hydrog. Energy 2021, 46, 28298-28326. [CrossRef]

39. Ullah, H.; Khan, I.; Fiza, M.; Hamadneh, N.N.; Fayz-Al-Asad, M.; Islam, S.; Khan, I.; Raja, M.A.Z.; Shoaib, M. MHD boundary layer flow over a stretching sheet: A new stochastic method. Math. Probl. Eng. 2021, 2021, 9924593. [CrossRef]

40. Ahmad, I.; Ilyas, H.; Raja, M.A.Z.; Khan, Z.; Shoaib, M. Stochastic numerical computing with Levenberg-Marquardt backpropagation for performance analysis of heat sink of functionally graded material of the porous fin. Surf. Interfaces 2021, 26, 101403. [CrossRef]

41. Khan, I.; Ullah, H.; AlSalman, H.; Fiza, M.; Islam, S.; Zahoor Raja, A.; Shoaib, M.; Gumaei, A.H. Falkner-Skan equation with heat transfer: A new stochastic numerical approach. Math. Probl. Eng. 2021, 2021, 3921481. [CrossRef]

42. Umar, M.; Sabir, Z.; Raja, M.A.Z.; Aguilar, J.G.; Amin, F.; Shoaib, M. Neuro-swarm intelligent computing paradigm for nonlinear HIV infection model with CD ${ }^{4+}$ T-cells. Math. Comput. Simul. 2021, 188, 241-253. [CrossRef]

43. Sabir, Z.; Raja, M.A.Z.; Guirao, J.L.; Saeed, T. Meyer Wavelet neural networks to solve a novel design of fractional order pantograph lane-emden differential model. Chaos Solitons Fractals 2021, 152, 111404. [CrossRef]

44. Ali, W.; Li, Y.; Raja, M.A.Z.; Khan, W.U.; He, Y. State estimation of an underwater markov chain maneuvering target using intelligent computing. Entropy 2021, 23, 1124. [CrossRef] [PubMed]

45. Shercliff, J.A. Textbook of Magnetohydrodynamics; Pergamon Press: Oxford, UK, 1965.

46. Cramer, K.R.; Pai, S.I. Magnetofluid Dynamics for Engineers and Applied Physicists; McGrawhill: New York, NY, USA, 1973.

47. Hayat, T.; Khan, S.A.; Alsaedi, A. Simulation and modeling of entropy optimized MHD flow of second grade fluid with dissipation effect. J. Mater. Res. Technol. 2020, 9, 11993-12006. [CrossRef]

48. Magyari, E.; Chamkha, A.J. Exact analytical results for the thermosolutal MHD Marangoni boundary layers. Int. J. Therm. Sci. 2008, 47, 848-857. [CrossRef]

49. Sheikholeslami, M.; Chamkha, A.J. Influence of Lorentz forces on nanofluid forced convection considering Marangoni convection. J. Mol. Liq. 2017, 225, 750-757. [CrossRef]

50. Shoaib, M.; Akhtar, R.; Khan, M.A.R.; Rana, M.A.; Siddiqui, A.M.; Zhiyu, Z.; Raja, M.A.Z. A novel design of three-dimensional mhd flow of second-grade fluid past a porous plate. Math. Probl. Eng. 2019, 2019, 2584397. [CrossRef]

51. Shoaib, M.; Rana, M.A.; Siddiqui, A.M. The effect of slip condition on the three-dimensional flow of Jeffrey fluid along a plane wall with periodic suction. J. Braz. Soc. Mech. Sci. Eng. 2017, 39, 2495-2503. [CrossRef] 
52. Siddiqui, A.M.; Shoaib, M.; Rana, M.A. Three-dimensional flow of Jeffrey fluid along an infinite plane wall with periodic suction. Meccanica 2017, 52, 2705-2714. [CrossRef]

53. Awais, M.; Raja, M.A.Z.; Awan, S.E.; Shoaib, M.; Ali, H.M. Heat and mass transfer phenomenon for the dynamics of casson fluid through porous medium over shrinking wall subject to Lorentz force and heat source/sink. Alex. Eng. J. 2021, 60, 1355-1363. [CrossRef] 